ARTICLE

Received 8 Jul 2015 | Accepted 28 Aug 2015 | Published 7 Oct 2015

DOI: 10.1038/ncomms9501

OPEN

\title{
A PI3K p110ß-Rac signalling loop mediates Pten-loss-induced perturbation of haematopoiesis and leukaemogenesis
}

\author{
Haluk Yuzugullu ${ }^{1,2, *}$, Lukas Baitsch ${ }^{1,2,}$, Thanh Von ${ }^{1,2}$, Allison Steiner ${ }^{1}$, Haoxuan Tong ${ }^{1,2}$, Jing Ni ${ }^{1,2}$, \\ Linda K. Clayton', Roderick Bronson ${ }^{3}$, Thomas M. Roberts ${ }^{1,2}$, Kira Gritsman ${ }^{1,2, \dagger}$ \& Jean J. Zhao ${ }^{1,2}$
}

The tumour suppressor PTEN, which antagonizes PI3K signalling, is frequently inactivated in haematologic malignancies. In mice, deletion of PTEN in haematopoietic stem cells (HSCs) causes perturbed haematopoiesis, myeloproliferative neoplasia (MPN) and leukaemia. Although the roles of the PI3K isoforms have been studied in PTEN-deficient tumours, their individual roles in PTEN-deficient HSCs are unknown. Here we show that when we delete PTEN in HSCs using the Mx1-Cre system, p110 $\beta$ ablation prevents MPN, improves HSC function and suppresses leukaemia initiation. Pharmacologic inhibition of p110 $\beta$ in PTEN-deficient mice recapitulates these genetic findings, but suggests involvement of both Akt-dependent and -independent pathways. Further investigation reveals that a p110 $\beta$-Rac signalling loop plays a critical role in PTEN-deficient HSCs. Together, these data suggest that myeloid neoplasia driven by PTEN loss is dependent on p110 $\beta$ via p110 $\beta$-Rac-positive-feedback loop, and that disruption of this loop may offer a new and effective therapeutic strategy for PTEN-deficient leukaemia.

\footnotetext{
${ }^{1}$ Department of Cancer Biology, Dana-Farber Cancer Institute, Boston, Massachusetts 02215, USA. ${ }^{2}$ Department of Biological Chemistry and Molecular Pharmacology, Harvard Medical School, Boston, Massachusetts 02215, USA. ${ }^{3}$ Dana-Farber/Harvard Cancer Center Rodent Histopathology Core, Harvard Medical School, Boston, Massachusetts 02215, USA. † Present address: Department of Medicine, Department of Cell Biology, Albert Einstein College of Medicine, Bronx, New York 10461, USA. * These authors contributed equally to this work. Correspondence and requests for materials should be addressed to T.M.R. (email: Thomas_roberts@dfci.harvard.edu) or to K.G. (email: kira.gritsman@einstein.yu.edu) or to J.J.Z. (email: jean_zhao@dfci.harvard.edu).
} 
$\mathrm{D}$ ysregulation of the molecular pathways involved in the self-renewal, differentiation and proliferation of haematopoietic stem cells (HSCs) can cause leukaemia. Notably, the serine/threonine kinase Akt, which acts downstream of PI3 kinase (PI3K), is hyper-phosphorylated in up to $80 \%$ of acute myeloid leukaemia (AML) cases ${ }^{1}$. This is unlikely to be due to mutations in upstream receptor tyrosine kinases alone. In chronic myelogenous leukaemia, PI3K/Akt signalling can also be activated through downregulation of the phosphatase and tensin homologue (PTEN) by BCR-ABL ${ }^{2}$. PTEN is a lipid phosphatase that counteracts PI3K signalling by dephosphorylating phosphatidylinositol-3,4,5-trisphosphate (PIP3). PTEN is frequently inactivated in haematological malignancies ${ }^{3,4}$, including in AML and $\mathrm{T}$ cell acute lymphoblastic leukemia (T-ALL) ${ }^{5}$. Notably, PTEN expression is often reduced in the disease through several other modes of PTEN regulation, for example, microRNAs, epigenetic modifications and ubiquitination ${ }^{6-9}$, which likely contribute to the high frequency of Akt phosphorylation in myeloid leukaemia.

In mice, genetic ablation of PTEN in the haematopoietic system leads to HSC depletion in the bone marrow (BM), myeloproliferative neoplasia (MPN) and transplantable acute leukaemia (myeloid or T-cell leukaemia) ${ }^{10-12}$. In patients, MPNs such as chronic myelogenous leukaemia or myelofibrosis can progress to $\mathrm{AML}^{13}$.

Class I PI3Ks are heterodimeric lipid kinases that produce the lipid second messenger PIP3 on stimulation of cells by many growth factors. Class I PI3Ks are divided into class IA (p110 $\alpha$, $\mathrm{p} 110 \beta$ and $\mathrm{p} 110 \delta)$ and class IB (p110 $\gamma$ ) enzymes; of these, the $\mathrm{p} 110 \alpha$ and $\mathrm{p} 110 \beta$ isoforms are ubiquitously expressed, while $\mathrm{p} 110 \delta$ and $\mathrm{p} 110 \gamma$ are enriched in leukocytes. Work in several different murine models has documented distinct requirements for different PI3K isoforms in particular tumour types ${ }^{14,15}$. For example, p1 $10 \alpha$ is essential in a model of mutant Kras-induced lung adenocarcinoma ${ }^{16}$. Recently, we showed that Ras-mutated myeloid leukaemia is also dependent on the p110 $\alpha$ isoform, and combined pharmacologic inhibition of $\mathrm{p} 110 \alpha$ and mitogenactivated protein kinase kinase (MEK) could be an effective therapeutic strategy for Ras-mutated myeloid malignancies ${ }^{17}$. Although p1 $10 \beta$ plays a less prominent role in receptor tyrosine kinase (RTK) signalling, it mediates $\mathrm{G}$ protein-coupled receptor (GPCR) and integrin signalling ${ }^{18-20}$, and has been shown to interact specifically with Rho family GTPases Rac1 and CDC42 (ref. 21 ). Several recent studies demonstrated that $\mathrm{p} 110 \beta$ is required in many, but not all, PTEN-deficient solid tumours ${ }^{20,22,23}$. However, it is not known which PI3K isoforms are most important for myeloid neoplastic transformation driven by PTEN loss.

A number of pan-class I PI3K and dual class I/mTOR inhibitors are now in clinical trials for cancer, including leukaemia. However, targeting PI3K with these inhibitors could potentially lead to severe toxicity, which could be prevented by targeting single PI3K isoforms. To this end, numerous isoform-selective compounds are currently under development with some already in clinical trials ${ }^{14}$. The p1108-selective inhibitor idelalisib (referred to here as GS1101) has been remarkably effective in treating indolent B-cell malignancies, and is now approved by the FDA for the treatment of chronic lymphocytic leukaemia ${ }^{24}$. In the case of solid tumours, p110 $\alpha$-selective inhibitors have shown great promise in early-phase trials for patients with tumours bearing PIK3CA mutations ${ }^{14}$. Notably, selective inhibitors of p110 $\beta$ are in clinical trials as anticancer reagents for advanced solid tumours with PTEN deficiency (NCT01458067). Thus, unravelling the role of each $\mathrm{PI} 3 \mathrm{~K}$ isoform, and its contribution to leukaemic transformation driven by PTEN loss, would inform rational approaches in targeting the PI3K pathway with a better therapeutic window.

In the present study, we used genetically engineered mouse models to determine which of the class IA PI3K isoforms are most important in mediating the effects of Pten loss in HSCs. We show that, in the setting of Pten loss, p110 $\beta$ is the main PI3K isoform responsible for MPN development and HSC depletion in the BM. Furthermore, we show that isoform-selective PI3K inhibitors recapitulate our genetic findings. We also found that a signalling loop featuring p110 $\beta$ and Rac plays an important role in the absence of Pten. Our results suggest that targeting p110 $\beta$ and/or Rac may lead to an effective therapeutic strategy for PTEN-deficient myeloid leukaemia.

\section{Results}

p110 $\beta$ ablation prolongs survival of Mx-1-Cre; ten $^{\mathrm{f} / \mathrm{fl}}$ mice. To explore the roles of the class IA PI3K isoforms in HSCs and leukaemic transformation in the absence of Pten, we crossed $\mathrm{Mx}-1-\mathrm{Cre}^{+} ; \mathrm{Pten}^{\mathrm{f} / \mathrm{fl}}$ mice $^{10,11}$ with strains that carry homozygous floxed alleles of p $110 \alpha$ (ref. 25) or p1 $10 \beta$ (ref. 20), or with a p110 $\delta$ germline knockout strain ${ }^{26}$ to allow for simultaneous deletion of Pten and PI3K isoforms in HSCs. As Mx1 is an interferonresponsive promoter expressed in HSCs and all other haematopoietic cells, the expression of Cre recombinase can be induced by administration of double-stranded RNA polyI-polyC (pIpC), which induces an interferon response ${ }^{10,27}$. Mx-1-Cre ${ }^{+}$; $\mathrm{Pten}^{\mathrm{f} / \mathrm{fl}}\left(\right.$ Pten $\left.^{\Delta / \Delta}\right), \quad \mathrm{Mx}-1-\mathrm{Cre}^{+} ; \mathrm{Pten}^{\mathrm{f} / \mathrm{fl}} ; \mathrm{p} 110 \alpha^{\mathrm{f} / \mathrm{fl}} \quad\left(\right.$ Pten $^{\Delta / \Delta}$; $\left.p 110 \alpha^{\Delta / \Delta}\right), \quad$ Mx-1-Cre ${ }^{+} ;$Pten $^{\mathrm{f} / / \mathrm{fl}} ;$ p110 $\beta^{\mathrm{fl} / \mathrm{fl}}{ }^{\left.; \text {Pten }^{\Delta / \Delta} ; p 110 \beta^{\Delta / \Delta}\right)}$ and $\mathrm{Mx}-1-\mathrm{Cre}^{+} ; \mathrm{Pten}^{\mathrm{f} / \mathrm{f}} ; \mathrm{p} 110 \delta^{\mathrm{KO} / \mathrm{KO}}\left(\right.$ Pten $^{\Delta / 4} ; \mathrm{p}^{110 \delta^{-/-}}{ }^{-}$mice were treated with $\mathrm{pIpC}$ to induce Cre-mediated excision of floxed alleles at 4 weeks of age (Supplementary Fig. 1a). This leads to the deletion of Pten and/or PI3K isoforms in haematopoietic cells, including HSCs or progenitor cells in the BM, spleen, thymus and liver ${ }^{11}$

Consistent with previous studies ${ }^{10,11}$, all $\mathrm{Pten}^{\Delta / \Delta}$ mice developed MPN and reached the survival end point 20-40 days post injection (DPI; Fig. 1a). Pten ${ }^{\Delta / \Delta} ; 110 \alpha^{\mathrm{S} / \Delta}$ and $\operatorname{Pten}^{\Delta / \Delta}$; p110 $\delta^{-/-}$mice also developed MPN with slightly extended survival (Fig. 1a). Notably, Pten ${ }^{\Delta / \Delta} ; p 110 \beta^{\Delta / \Delta}$ mice lived the longest, with median survival significantly longer than that of any other group (Fig. 1a). Further observation revealed that, whereas control, Pten ${ }^{4 / \Delta}$, Pten $^{\Delta / \Delta} ; p 110 \alpha^{\Delta / \Delta}$ and Pten ${ }^{\Delta / \Delta} ; p 110 \delta^{-1-}$ animals developed MPN, six out of nine Pten ${ }^{\Delta / \Delta} ; p 110 \beta^{\Delta / \Delta}$ mice succumbed to T-ALL (Fig. 1b). BM from the three Pten ${ }^{\Delta / \Delta}$; $p 110 \beta^{\Delta / \Delta}$ mice that did develop MPN was analysed for excision of the pik3cb allele. Notably, we found that BM of these mice had incomplete deletion of Pik $3 \mathrm{cb}$ alleles suggesting that $\mathrm{p} 110 \beta$ is critical for the development of MPN in this model (Supplementary Fig. 1b). Deletion of p110 $\beta$ in HSCs using Mx1-Cre in animals that are wild-type (WT) for Pten does not significantly affect blood counts (Supplementary Fig. 1c).

Histopathological analysis of moribund animals of $\mathrm{Pten}^{4 / \Delta}$ Pten $^{\Delta / \Delta} ; p 110 \alpha^{\Delta / 4}$ and Pten ${ }^{\Delta / 4} ; p 110 \delta^{-1-}$ mice at $20-40$ days post PIpC showed that they developed massive splenomegaly with a marked increase in cells expressing myeloperoxidase, a marker used to detect leukaemic cells of the myeloid lineage, in both the spleen and liver (Supplementary Fig. 2a), confirming MPN development in these mice. Notably, thymuses in these moribund mice appeared normal (Supplementary Fig. 2b). However, most Pten $^{\Delta / \Delta} ; p 110 \beta^{\Delta / 4}$ animals became moribund at 50-70 days post pIpC with markedly increased thymus weights (Supplementary Fig. 2b), infiltration of terminal deoxynucleotidyl transferasepositive T lymphoblasts $\left(\mathrm{CD} 4{ }^{+}\right.$or $\mathrm{CD} 4$ and $\mathrm{CD} 8$ double-positive T-cell blasts) in the thymus and BM and increased white blood cell counts (Supplementary Fig. 2a,c-e), all of which are manifestations of T-ALL. These results suggest that the p110 $\beta$ isoform of PI3K plays a uniquely important role in driving myeloid neoplastic transformation in mice with Pten-deficient HSCs, but does not contribute to the development of T-ALL in this murine model. 
a

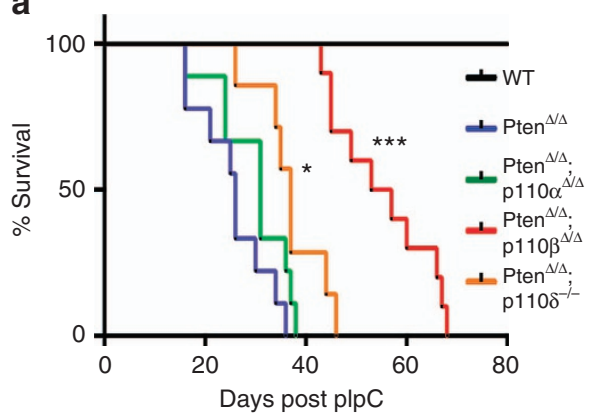

b

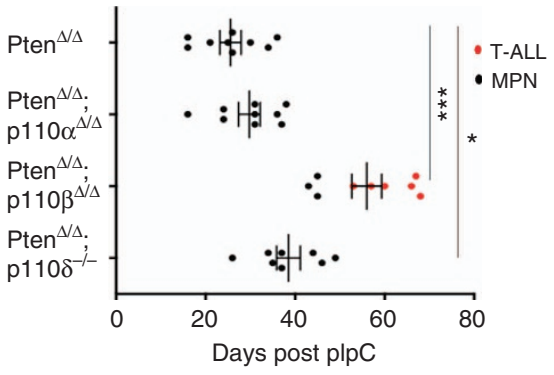

C
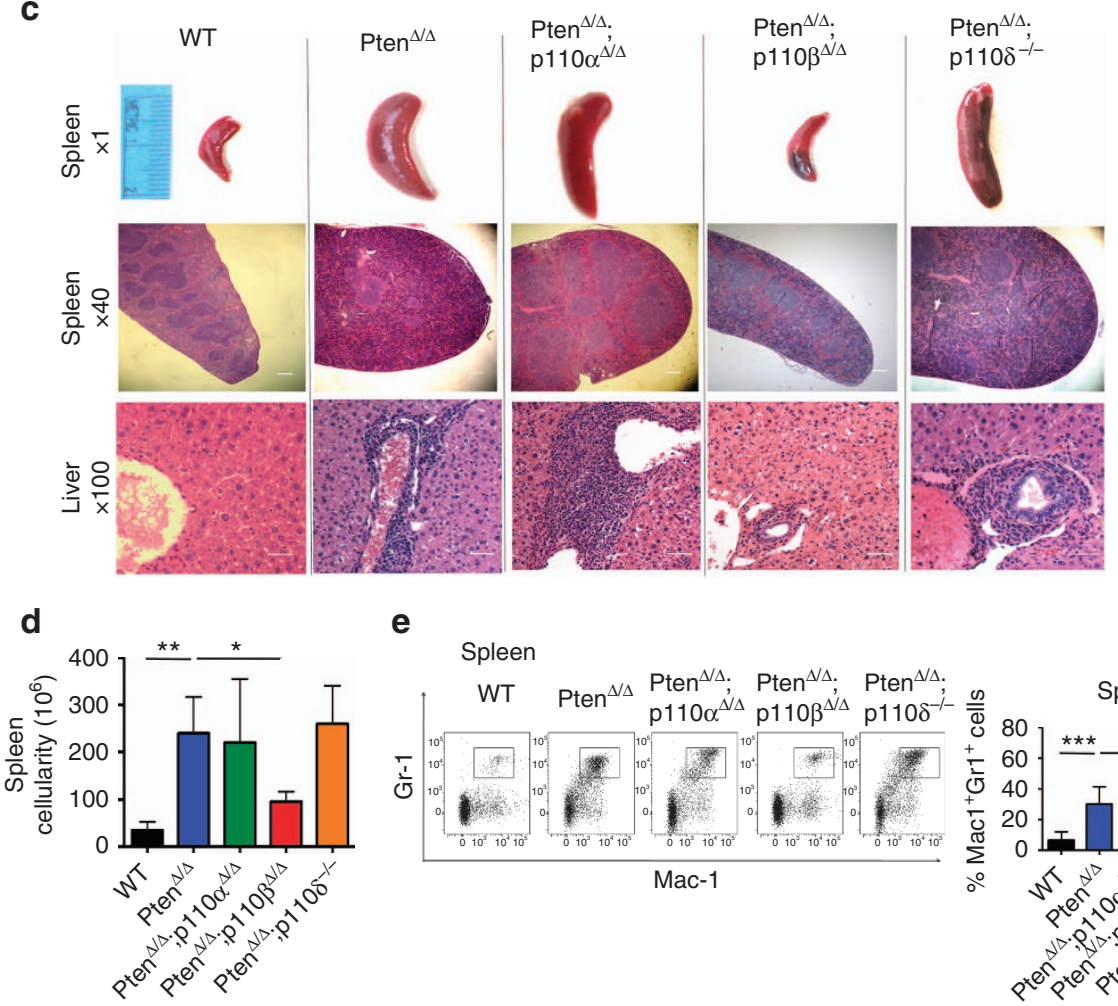

e


Figure 1 | Ablation of p110 $\beta$ prolonged survival of animals on Pten loss. (a) Survival of $P \operatorname{ten}{ }^{\Delta / \Delta}$ (blue; $n=9 ;$ median survival $=26$ days), Pten ${ }^{\Delta / \Delta} ; p 110 \alpha^{\Delta / \Delta}$ (green; $n=9 ;$ median survival = 31 days), $P \operatorname{ten}{ }^{\Delta / \Delta} ; p 110 \beta^{\Delta / \Delta}$ (red; $n=9 ;$ median survival $=55$ days), Pten ${ }^{\Delta / \Delta} ; p 110 \delta^{-/}-$(orange; $n=7 ;$ median survival $=37$ days) and control animals (black) represented as DPI. The log-rank test was used to compare survival between groups. (b) Survival time and the development of MPN and T-ALL in each group of mice as indicated. Survival of $\operatorname{Pten}^{\Delta / \Delta}\left(n=9\right.$; median survival $=26$ days), Pten ${ }^{\Delta / \Delta} ;$; $110 \alpha^{\Delta / \Delta}(n=9$; median survival $=31$ days $), \operatorname{Pten}^{\Delta / \Delta} ;$ p $110^{\Delta / \Delta}(n=9 ;$ median survival $=55$ days $), \operatorname{Pten}^{\Delta / \Delta} ;$ p $110 \delta^{-/}-(n=7 ;$ median survival $=37$ days $)$ and control animals (black) represented as DPI. Black dots represent MPN disease cases and red dots represent T-ALL cases in each group. The log-rank test was used to compare survival between groups. (c) Histopathology of the spleen $(\times 1$ and $\times 40)$ and liver $(\times 100)$ taken at 26 DPI. Increased splenomegaly and disruption of spleen architecture as well as increased myeloid cell infiltration after Pten deletion. Scale bar, $5 \mu \mathrm{m}$. (d) Total cellularity of the spleen is represented as mean \pm s.d. of $n=5$ except Pten ${ }^{\Delta / 4} ; p 110 \delta^{-/-}(n=10)$. Two-way ANOVA test was applied to compare the spleen cellularity. (e)

Representative flow cytometry plots of $\mathrm{Mac1}^{+} \mathrm{Gr}^{+}$cells in the spleen. Samples were analysed at $26 \mathrm{DPI}$. Bar graphs represent the mean \pm s.d. of $n=9$ $\left(\operatorname{Pten}^{\Delta / \Delta} ; p_{110 \delta^{-/-}}\right), 10\left(\operatorname{Pten}^{\Delta / \Delta} ; p 110 \alpha^{\Delta / \Delta}\right), 11(\mathrm{ctrl})$ or $13\left(\operatorname{Pten}^{\Delta / \Delta}\right.$ and Pten $\left.{ }^{\Delta / \Delta} ; p 110 \beta^{\Delta / \Delta}\right)$. Two-way ANOVA test was applied to compare the Mac1-Gr1 cells in the spleen. ${ }^{\star} P<0.05,{ }^{\star \star} P<0.01,{ }^{\star \star \star} P<0.001$

Previous studies have shown that Pten deletion in T-cell progenitors causes malignant transformation in the thymus and leads to T-cell lymphoma/T-ALL within 50-150 days ${ }^{28,29}$. Mice with Mx1-Cre-mediated deletion of Pten ${ }^{\Delta / 4}$, Pten $^{\Delta / \Delta} ;$ p110 $\alpha^{\Delta / \Delta}$ or $\operatorname{Pten}^{\Delta / \Delta} ;$ p $110 \delta^{-/-}$showed infiltrating MPN disease and became moribund within 20-40 days after pIpC, earlier than the disease latency for T-cell disease development. Since Pten ${ }^{\Delta / \Delta} ; p 110 \beta^{\Delta / \Delta}$ mice did not develop MPN, they survived longer and developed T-cell lymphoma/T-ALL 50-70 days post pIpC injection, a timeline consistent with previous reports on T-cell lymphoma/ T-ALL formation in models of Pten loss in T-cell progenitors ${ }^{28,29}$.
This suggests that $\mathrm{p} 110 \beta$ ablation does not prevent T-ALL formation driven by Pten loss.

To examine further the PI3K isoform dependence in T-ALL induced by Pten loss, we investigated the roles of p110 $\alpha$ and $p 110 \beta$ in a different T-ALL model driven by Pten ablation in T-cell progenitors using $\mathrm{Lck}_{-\mathrm{Cre}}{ }^{30}$. Interestingly, deletion of either $\mathrm{p} 110 \alpha$ or $\mathrm{p} 110 \beta$ had no effect on T-ALL in this model 3 (Supplementary Fig. 2f). These results underscore the distinct roles of p110 $\beta$ in myeloid and lymphoid neoplasia induced by Pten deletion in haematopoietic cells. 
p110 $\beta$ mediates myeloid expansion induced by Pten loss. To further characterize disease in the $\operatorname{Pten}^{\Delta / \Delta} ; p 110^{\Delta / \Delta}$ mice, we killed mice of each genotype at $26 \mathrm{DPI}$, the time point at which the $\operatorname{Pten}^{\Delta / \Delta}$ mice become moribund. Similar to previous reports, all animals in the $\mathrm{Pten}^{\Delta / \Delta}$ group displayed massive splenomegaly, increased spleen cellularity and loss of spleen architecture at this time point (Fig. 1c,d). Both Pten ${ }^{\Delta / \Delta} ; p_{110} \alpha^{\Delta / \Delta}$ and Pten ${ }^{\Delta / \Delta}$; $p 110 \delta^{-/-}$mice showed evidence of MPN similar to that of $P_{t e n}{ }^{1 / \Delta}$ mice, suggesting that ablation of $\mathrm{p} 110 \alpha$ or $\mathrm{p} 110 \delta$ failed to rescue this disease phenotype. Notably, Pten $^{\Delta / \Delta} ; p 110 \beta^{\Delta / \Delta}$ mice had significantly reduced spleen cellularity and size, compared with Pten $^{\Delta / \Delta}$ mice (Fig. 1c,d). Consistently, pathological analysis of the spleen and liver revealed infiltration of myeloid cells in Pten $^{\Delta / \Delta}$, Pten ${ }^{\Delta / \Delta} ; p 110 \alpha^{\Delta / \Delta}$ and Pten ${ }^{\Delta / \Delta} ; p 110 \delta^{-/-}$animals, but not in Pten $^{\Delta / \Delta} ;$ p110 $\beta^{\Delta / \Delta}$ mice (Fig. 1c). Flow cytometric analysis confirmed an increased population of myeloid cells $\left(\mathrm{Mac1}^{+}\right.$ $\left.\mathrm{Gr}^{+}\right)$in the BM, spleen and peripheral blood of $\operatorname{Pten}^{\Delta / \Delta}$ animals (Fig. 1e; Supplementary Fig. 3). Again, the numbers of $\mathrm{Macl}^{+}$ $\mathrm{Gr}^{+}{ }$cells in these organs in Pten $^{\Delta / \Delta} ; p 110 \beta^{\Delta / \Delta}$ but not in

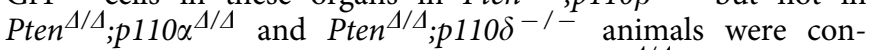
sistently reduced compared with those of $\operatorname{Pten}^{\Delta / \Delta}$ mice (Fig. 1e; Supplementary Fig. 3), suggesting that ablation of p110 suppressed myeloid cell expansion on Pten loss.

To further validate the role of $\mathrm{p} 110 \beta$ in the myeloid expansion caused by Pten loss, we performed colony assays in methylcellulose supplemented with myeloid growth factors. Compared with wild-type controls, both BM and spleen cells from Pten ${ }^{4 / \Delta}$ animals generated an increased number of colonies, which was significantly reduced in $P t e n^{\Delta / \Delta} ; p 110 \beta^{\Delta / \Delta}$ mice (Fig. 2a). Together, these results demonstrate that $\mathrm{p} 110 \beta$ is required for MPN development in the absence of Pten.

To determine whether the contribution of $\mathrm{p} 110 \beta$ to myeloid neoplasia in the absence of Pten is a cell-autonomous or indirect effect, we transplanted whole BM cells from $\operatorname{Pten}^{\Delta / \Delta}, \operatorname{Pten}^{\Delta / \Delta}$; $p 110 \beta^{\Delta / \Delta}$ or control mice into recipient mice (Fig. 2b). Four weeks after transplantation, all groups were treated with $\mathrm{pIpC}$, and the relative frequency of $\mathrm{CD} 45.2^{+}$donor-derived $\mathrm{Mac1}{ }^{+}$ $\mathrm{Gr}^{+}$cells was monitored over 16 weeks. The proportion of Pten $^{4 / \Delta}$ donor-derived myeloid cells expanded significantly 4 weeks after $\mathrm{pIpC}$ and remained elevated during the course of the experiment. In contrast, the $P t e n^{\Delta / \Delta} ; p 110 \beta^{\Delta / \Delta}$ donor-derived myeloid population remained stable during the entire experiment, with levels much comparable to that of wild-type control mice (Fig. 2c). There were no significant differences in the percentage of donor-derived CD3-positive T cells among any of the groups tested (Supplementary Fig. 4a). The percentage of donor-derived B220-positive B cells was reduced after Pten deletion, and deletion of $\mathrm{p} 110 \beta$ did not alter B-cell chimaerism (Supplementary Fig. $4 \mathrm{~b}$ ). These data suggest that $\mathrm{p} 110 \beta$ mediates the expansion of myeloid cells in a cell-autonomous manner.

p110ß perturbs HSC homeostasis on loss of Pten. Earlier studies showed that HSC-specific deletion of Pten leads to the exhaustion of HSCs in the BM, their accumulation in the periphery and extramedullary haematopoiesis ${ }^{10,11}$. Hence, we wanted to test whether $\mathrm{p} 110 \beta$ ablation could rescue HSCs. In $\operatorname{Pten}^{\Delta / \Delta}$ mice, the numbers of both the $\mathrm{Lin}^{-} \mathrm{Sca}-1^{+} \mathrm{c}$-kit ${ }^{+}$(LSK) cells, containing HSCs and the $\mathrm{CD} 150^{+} \mathrm{CD}_{4} 8^{-} \mathrm{Lin}^{-} \mathrm{Sca}-1^{+} \mathrm{c}_{\mathrm{kit}}{ }^{+}$population, which is enriched for long-term HSCs (LT-HSCs) were significantly reduced at $26 \mathrm{DPI}$, consistent with previous findings $^{11}$ (Fig. 3a; Supplementary Fig. 5a). Ablation of p110 was able to partially rescue LSK cells and restore LT-HSCs in Pten-null BM (Fig. 3a). Loss of Pten did not change the total number of myeloid progenitors, or the frequencies of the common myeloid progenitors and granulocyte macrophage progenitors or megakaryocyte-erythroid progenitors, but led to a significant decrease in the number of common lymphoid progenitors consistent with original reports ${ }^{11}$ (Supplementary

a


C

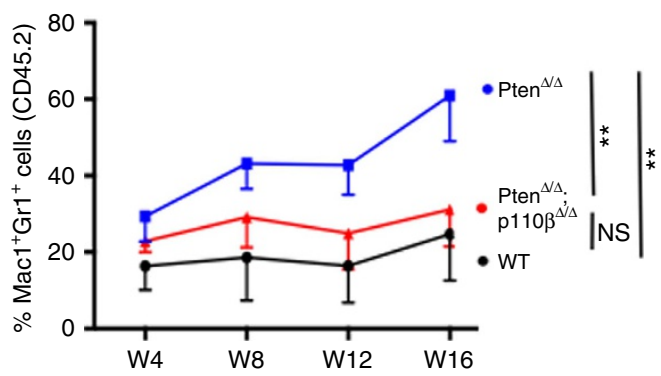

Weeks post transplantation

Figure 2 | Ablation of p110 $\beta$ blocked MPN development in a cell-autonomous manner. (a) Colony-forming assays were performed with $1 \times 10^{4}$ (BM) or $5 \times 10^{4}$ (spleen) cells obtained from corresponding animals. Cells were cultured in M3434 medium. Colonies were counted 7 days after incubation. Data are represented as mean \pm s.e.m. ( $n=3$, in duplicates). ${ }^{\star} P<0.05,{ }^{\star \star} P<0.01,{ }^{\star \star \star} P<0.001$. (b) BM cells were isolated from CD45.2 ${ }^{+} W T, P t e n^{\Delta / \Delta}$ and $P \operatorname{Pten}{ }^{\Delta / \Delta} ; p 110 \beta^{\Delta / \Delta}$ animals and transplanted into CD45.1 ${ }^{+}$mice as shown in the figure ( $n=10$ for each group). (c) The myeloid cell reconstitution potential of the CD45.2 $2^{+}$cells was measured at regular intervals for 16 weeks. WT and $P \operatorname{ten}{ }^{\Delta / \Delta} ;$ p $110 \beta^{\Delta / \Delta}$-derived myeloid cells have a stable amount of CD45.2 ${ }^{+}$ myeloid cells; however, $P$ ten ${ }^{\Delta / \Delta}$-derived myeloid cells expanded significantly and reached to $60 \%$ at week $16\left(n=10\right.$ for all groups, $\left.{ }^{\star \star} P<0.001\right)$. Two-way ANOVA test was applied to compare the myeloid cell potential. 


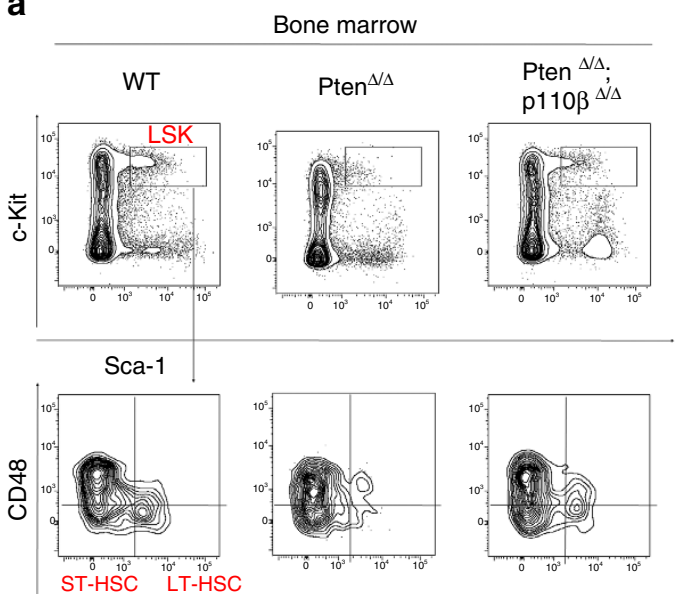

CD150

b

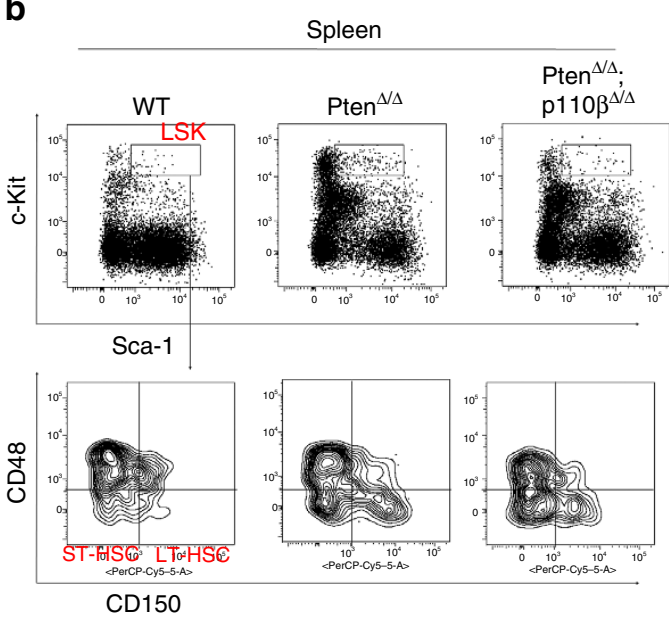

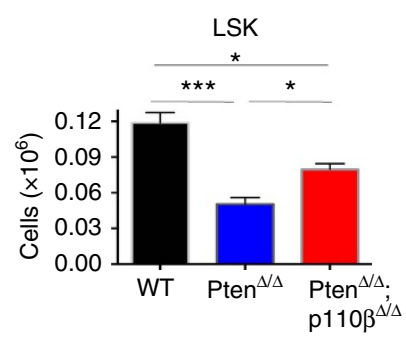

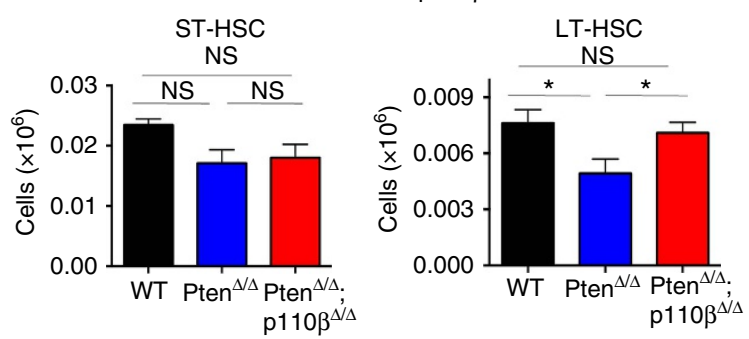


a

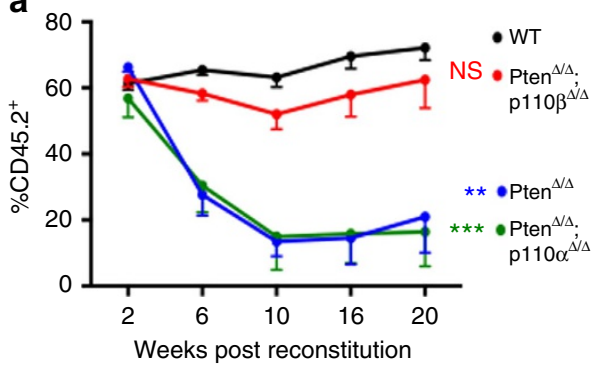

b

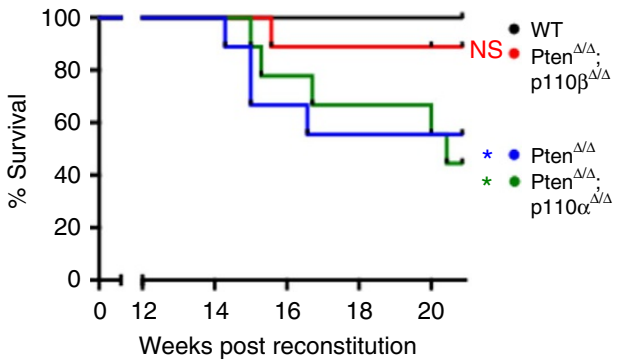

C
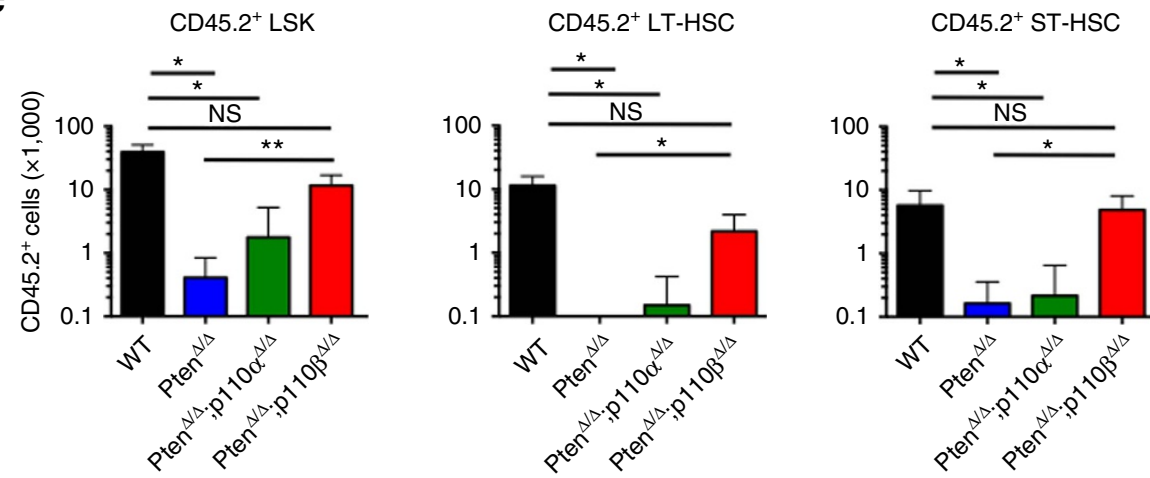

d

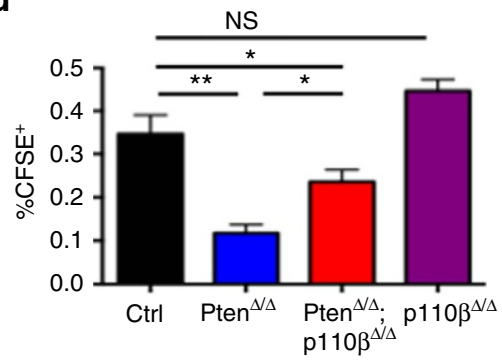

Figure 4 | p110 $\beta$ deletion rescued long-term HSC (LT-HSC) reconstitution and suppressed leukaemia initiation and homing defects after Pten deletion. (a) Competitive repopulation experiment: bone marrow (BM) cells were isolated at 7 days post plpC (DPI) from CD $45.2^{+} \mathrm{ctrl}_{1} P \operatorname{Pten}{ }^{\Delta / \Delta}, \mathrm{Pten}^{\Delta / \Delta} ; \mathrm{p} 110 \alpha^{\Delta / \Delta}$ and $P \operatorname{ten}{ }^{\Delta / \Delta} ; p 110 \beta^{\Delta / \Delta}$ animals, and transplanted into lethally irradiated recipient animals along with CD45.1 ${ }^{+}$BM cells. The donor chimaerism in the peripheral blood was measured at regular intervals for 20 weeks, represented as the percentage of $\mathrm{CD} 45.2^{+}$cells among all leukocytes in the peripheral blood ( $n=9$ for all groups). Two-way ANOVA test was applied to compare the donor derived cell numbers. (b) Disease-free survival of animals in the competitive repopulation experiment. Statistical analysis was performed at week 20 post transplantation using Fisher's exact test. All samples were compared with the control group. Fischer exact test was applied to test overall survival. (c) After 20 weeks, the competitive repopulation experiment was terminated, and the remaining numbers of LSK, ST-HSC and LT-HSCs in the BM were measured by flow cytometry $(n=4$ for each group except $n=5$ for $\operatorname{Pten}^{\Delta / \Delta} ; \mathrm{p}_{110 \delta^{-/-}}$). Two-way ANOVA test was applied to compare the donor derived stem cell numbers. (d) Homing potential of CD45.2-positive carboxyfluorescein diacetate succinimidyl ester (CFSE)-labelled control, $P \operatorname{ten}{ }^{\Delta / \Delta}$ and Pten ${ }^{\Delta / \Delta} ; p 110 \beta^{\Delta / \Delta}$ cells in $24 \mathrm{~h}$ in irradiated recipients $(n=5)$ for each group. Two-way ANOVA test was applied to compare the donor derived stem cell numbers. ${ }^{\star} P<0.05$, ${ }^{\star \star} P<0.01,{ }^{\star \star \star} P<0.001$

HSCs derived from $P t e n^{\Delta / 4} ; p 110 \beta^{\Delta / \Delta}$ mice were able to reconstitute recipient animals for more than 20 weeks (Fig. 4a). In addition, the majority of Pten $n^{\Delta / 4}$ and $P t e n^{\Delta / 4} ; p 110 \alpha^{\Delta / 4}$-recipient mice developed T-ALL as evidenced by the abundance of donor-derived CD $45.2^{+}$ $\mathrm{CD} 3{ }^{+} ; \mathrm{CD} 4{ }^{+}$or $\mathrm{CD} 3^{+} \mathrm{CD} 4^{-} \mathrm{T}$ lymphoblasts at the experimental end point of 20 weeks in these mice (Fig. 4b; Supplementary Fig. 6). In contrast, recipients of BM from wild-type control mice and from the majority of $P \operatorname{ten}{ }^{\Delta / \Delta} ; p 110 \beta^{\Delta / \Delta}$ animals remained leukaemia free with few $\mathrm{CD} 45.2^{+} \mathrm{CD}^{+}$cells at the experimental end point (Fig. 4b; Supplementary Fig. 6). Furthermore, analysis of the $\mathrm{BM}$ at week 20 showed that the donor chimaerism in the LSK, ST-HSC and LT-HSC compartments was significantly improved in the Pten ${ }^{\Delta / \Delta}$ and Pten $^{\Delta / \Delta} ; p 110 \alpha^{\Delta / \Delta}$ groups (Fig. 4c). These data suggest that, in the absence of Pten, $\mathrm{p} 110 \beta$ is the major PI3K isoform critical for the loss of HSCs and for leukaemia initiation.
To determine the cellular mechanism underlying the improved reconstitution of Pten-null BM cells on loss of p110 $\beta$, we examined the cell cycle status, senescence, apoptosis and homing properties of HSCs. As reported earlier, we also found that loss of Pten led to increased cycling of HSCs and reduced the number of quiescent HSCs (Supplementary Fig. 7a ${ }^{11}$. Although ablation of p110 $\beta$ resulted in a tendency towards rescuing these effects, the results did not reach statistical significance (Supplementary Fig. 7a). Moreover, we did not observe any change in the proportion of whole BM or LSK cells expressing senescenceassociated $\beta$-gal activity or undergoing apoptosis in any of the groups tested (Supplementary Fig. 7b,c). We then performed homing assays, in which fluorescently labelled BM cells were transplanted into irradiated wild-type hosts, and donor-derived cells were quantified after $24 \mathrm{~h}$. We found that Pten deficiency significantly reduced the homing capacity of transplanted cells to 
the $\mathrm{BM}$, and $\mathrm{p} 110 \beta$ ablation could partially rescue the homing potential (Fig. 4d). We also found that p110 $\beta$ ablation does not affect homing in Pten-wild-type BM cells (Fig. 4d). Thus, we conclude that $\mathrm{p} 110 \beta$ is responsible, at least in part, for the reduced homing activity of Pten-deficient HSCs.

Inhibition of p110 suppresses myeloid leukaemogenesis. To determine whether our findings using a genetic method could be recapitulated by pharmacologic approaches utilizing PI3K isoform-selective inhibitors at effective doses as published in earlier studies, we first examined the effects of PI3K isoform inhibition on myeloid progenitor function. We cultured BM cells and splenocytes from Pten ${ }^{\Delta / \Delta}$ animals in methylcellulose supplemented with myeloid growth factors in the presence of PI3K inhibitors. As reported in previous studies ${ }^{10}$, the pan-PI3K inhibitor GDC0941 and the mTOR inhibitor RAD001 significantly suppressed the increased colony formation arising from Pten deletion in a dosedependent manner (Fig. 5a). Consistent with our genetic findings, inhibition of p110 $\alpha$ with BYL719 (a p110 $\alpha$-selective inhibitor) ${ }^{32}$ and p1108 with GS1101 (ref. 33) had a modest effect on the expansion of myeloid cells in the context of Pten loss (Fig. 5a). Since $\mathrm{p} 110 \gamma$ is also expressed in leukocytes, we tested the p110 $\gamma$ inhibitor NVSPI35 (ref. 34). Interestingly, inhibition of p110 $\gamma$ with NVSPI35 showed some effect on BM cells, but not on splenocytes (Fig. 5a). Notably, inhibition of p110 $\beta$ with KIN193 (a p110ßselective inhibitor, also known as AZD6482) ${ }^{14,22}$ significantly reduced formation of both $\mathrm{BM}$ - and spleen-derived myeloid colonies in methylcellulose in a dose-dependent manner (Fig. 5a) suggesting that pharmacologic inhibition of p110 $\beta$ is highly effective in suppressing myeloid cell expansion driven by Pten loss.

To further determine the effects of pharmacologic inhibition of PI3K isoforms in vivo, we treated a group of $\mathrm{pIpC}$-induced Pten $^{\Delta / \Delta}$ animals with BYL719, KIN193, IC87114 (a p1108selective inhibitor in the same class as GS1101, but with better bioavailability in mice $)^{35}$, AS605240 (p110 $\gamma$-selective inhibitor suitable for in vivo studies) ${ }^{36}$ or a vehicle control 10 days after induction (Fig. 5b). All the inhibitors were used at the effective doses in vivo as published in earlier studies ${ }^{36-38}$. Treatment with BYL719 (ref. 37), IC87114 (ref. 38) or AS605240 (ref. 36) resulted in a minimal survival benefit compared with vehicle-treated animals (Fig. 5c). Notably, mice treated with KIN193 had a significantly longer survival as compared with mice in any other group (Fig. 5c). Moreover, KIN193-treated animals appeared healthy and had significantly reduced spleen weights and normallooking spleen architecture compared with vehicle-treated mice (Fig. 5d; Supplementary Fig. 8), consistent with our genetic data that ablation of p110 $\beta$ largely prevented myeloid leukaemia in Pten-deficient mice.

Next, we examined PI3K/Akt signalling in Pten-null BM cells in response to isoform-selective inhibition. As expected, vehicletreated Pten-null BM cells from Pten ${ }^{\Delta / \Delta}$ animals showed markedly increased Akt phosphorylation compared with wild-type BM cells (Fig. 5e). Interestingly, inhibition of either $\mathrm{p} 110 \alpha, \mathrm{p} 110 \beta$ or $\mathrm{p} 110 \delta$ led to a significant reduction of $\mathrm{p}-\mathrm{Akt}$, compared with the controls (Fig. 5e). We also examined Akt activation by measuring basal p-Akt levels in LSK cells by intracellular phosopho-flow cytometry after Pten deletion and short-term isoform-selective inhibitor treatment of lineagenegative cells from $\operatorname{Pten}^{\Delta / \Delta} \mathrm{BM}$. We detected significantly higher basal levels of p-Akt in Pten ${ }^{4 / 4}$ LSK cells compared with WT LSKs, and again inhibition of $\mathrm{p} 110 \alpha, \mathrm{p} 110 \beta, \mathrm{p} 110 \delta$ or $\mathrm{p} 110 \gamma$ led to a significant reduction of p-Akt compared with the vehicle group (Fig. 5f). Since p110 $\beta$ is not the only isoform responsible for mediating Akt signalling in Pten-deficient $\mathrm{BM}$ and HSCs, Akt signalling alone is not sufficient to explain the specific biological effects of $\mathrm{p} 110 \beta$ ablation or inhibition observed in our study.

Pten-deficient HSCs depend on the p110ß-Rac axis. Recent data suggested that $\mathrm{p} 110 \alpha, \mathrm{p} 110 \delta$ and $\mathrm{p} 110 \gamma$ bind to and are activated by the Ras subfamily of GTPases, while p1 $10 \beta$ instead binds to and is activated by the Rho subfamily GTPases, Rac1 and CDC42 via its 'Ras-binding domain' (RBD) ${ }^{17,21}$. Previous studies also reported that an intact RBD was required for signalling and oncogenic transformation by wild-type $\mathrm{p} 110 \beta$, suggesting a potential role for the interaction of Rho GTPase with $\mathrm{p} 110 \beta$ in transformation ${ }^{39,40}$. Notably, Rac1 and CDC42 can also be activated downstream of PI3K by PIP3-dependent guaninenucleotide exchange factors ${ }^{41,42}$. It has been previously reported that Rac plays important roles in the homing and survival of $\mathrm{HSCs}^{43,44}$. Given the significant rescue of HSCs in $\mathrm{Pten}^{4 / 4}$; $p 110 \beta^{\Delta / \Delta}$ mice, we hypothesized that a unique positive-feedback signalling loop might exist between $\mathrm{p} 110 \beta$ and Rac, in which p110 $\beta$ is activated by Rac and Rac could in turn be activated by the phosphoinoside products of $\mathrm{p} 110 \beta$ in the setting of Pten-null haematopoietic cells.

Notably, we detected higher levels of Rac-GTP in the BM of $\mathrm{Pten}^{\mathrm{A} / \Delta}$ mice, which could be suppressed by deletion of $\mathrm{p} 110 \beta$ but not $\mathrm{p} 110 \alpha$ (Fig. 6a). To investigate whether the binding of p110 $\beta$ to Rac is important in mediating p110 $\beta$ activity in Ptendeleted BM cells, we mutated the two highly conserved key residues within the p110 $\beta$ RBD to generate a p110 $\beta-S 205 \mathrm{D} /$ K224A double mutant lacking the binding activity to Racl (ref. 21), and performed an add-back experiment with either wild-type or RBD-mutant $\mathrm{p} 110 \beta$ in BM cells derived from Pten ${ }^{\Delta / 4} ; p 110 \beta^{\Delta /}$ ${ }^{\Delta}$ mice (Fig. 6b; Supplementary Fig. 10). Colony-forming assays revealed that, while adding back a wild-type p110 $\beta$ in Pten $^{\Delta /}$ ${ }_{;} ; p 110 \beta^{4 / \Delta} \mathrm{BM}$ cells restored myeloid colony numbers comparable to those of $\operatorname{Pten}^{\Delta / \Delta} \mathrm{BM}$ cells, the RBD-mutant $\mathrm{p} 110 \beta$ failed to rescue colony formation (Fig. 6c). To determine whether p110 $\beta$ affects Rac-GTP levels in HSCs/progenitor cells (HSPCs), we performed the Rac-GTP assay either on Lin-negative Pten ${ }^{\Delta / \Delta}$ BM cells or Pten $^{\Delta / \Delta} ; p 110 \beta^{\Delta / \Delta}$ cells expressing wild-type $110 \beta$ and RBD-mutant p1 10 $\beta$. We detected higher levels of Rac-GTP in the Pten-deficient cells compared with WT control cells, and these levels were significantly reduced in $\operatorname{Pten}^{\Delta / \Delta} ; \mathrm{p}_{110} \beta^{\Delta / \Delta}$ cells. Adding back wild-type p110 $\beta$ to Pten $^{\Delta / 4} ;$ p $110 \beta^{4 / \Delta} \mathrm{HSC} / \mathrm{P}$ cells, partially rescued Rac-GTP levels but adding back RBD-mutant p110 $\beta$ failed to rescue Rac activity (Fig. 6d; Supplementary Fig. 10) Together, these data suggest that the interaction of p110 $\beta$ with Rac plays an important role in mediating the myeloid clonogenic activity driven by Pten loss.

To further investigate the functional dependency of Pten-deleted leukaemic cells on the p110 $\beta-$ Rac axis, we utilized NSC23766, a potent Rac inhibitor ${ }^{45}$ in our Pten-null model (Fig. 7a). We found that treatment of these mice for 10 days led to a reduced disease burden, as demonstrated by reduced spleen size and cellularity (Fig. 7a; Supplementary Fig. 9). Similarly, treatment of $\mathrm{Pten}^{4 / 4}$ mice with NSC23766 resulted in a significant reduction of HSC and myeloid progenitor numbers in the spleen compared with vehicle controls (Fig. 7b; Supplementary Fig. 9). Treatment with NSC23766 also led to a significantly prolonged survival of Pten ${ }^{4 / 4}$ mice (Fig. 7c), recapitulating the findings for genetic ablation or pharmacological inhibition of p110 $\beta$ in Pten-null animals.

Since Rac is required for $\mathrm{p} 110 \beta$ activation downstream of $\mathrm{GPCRs}^{21}$, we assessed the functional importance of the Rac-p110 $\beta$ signalling axis in HSPC function in response to activation of CXCR4, a GPCR important in the regulation of HSPCs. We used Transwell migration assays with CXCL12, a 
a


b

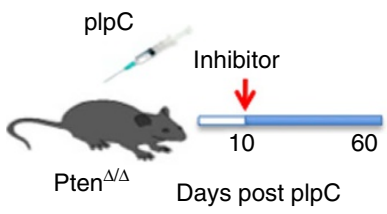

d

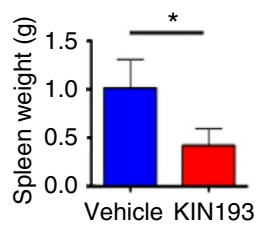

f

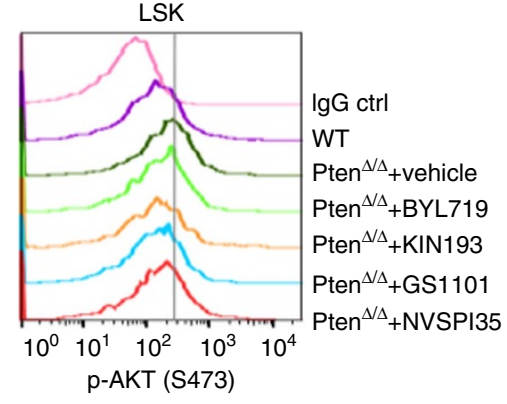

c.

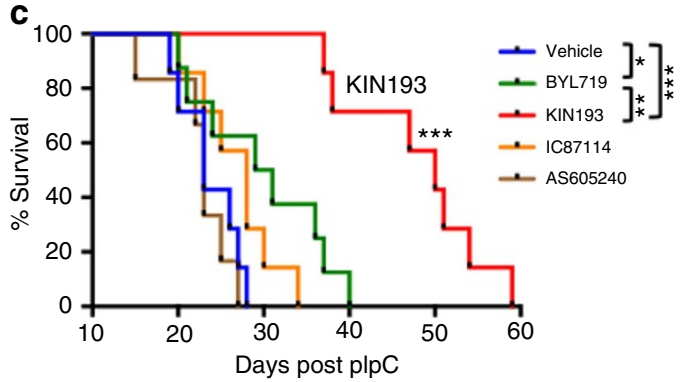

e

W WBM

$\operatorname{Pten}^{\Delta / \Delta}$

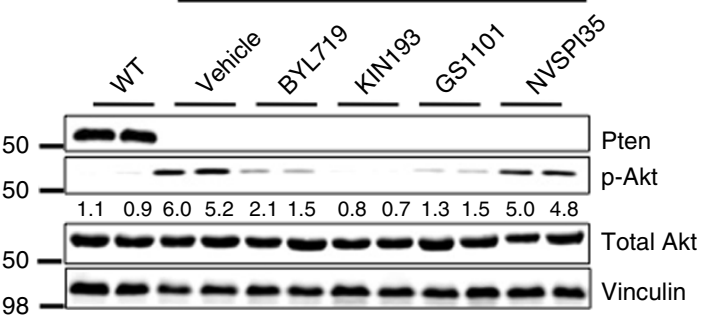



Figure 5 | Pharmacological inhibition of p110 $\beta$ suppresses leukaemia in vitro and in Pten-deficient mice. (a) Colony-forming assays were performed with $1 \times 10^{4}(\mathrm{BM})$ or $5 \times 10^{4}$ (spleen) cells obtained from 7 DPI Pten ${ }^{4 / \Delta}$ animals. Cells were cultured with added DMSO, BYL719, TGX221,GS1101, NVSPI35, GDC0941 or RAD001 with the following concentrations based on published data: 1, 0.5, $0.1 \mu \mathrm{M}$ (BYL719, TGX221 and GDC0941); 5, 1, 0.5 $\mu$ M (GS1101); $0.1,0.05,0.01 \mu \mathrm{M}$ (NVSPI35 and RAD001). Colonies were counted 7 days after incubation. Data are represented as mean \pm s.e.m. $(n=3$, measured in duplicates) and all data are compared with the DMSO control for statistical analysis. ${ }^{\star} P<0.05$; ${ }^{\star \star} P<0.001$. Two-way ANOVA test was applied to compare the colony formation. (b) Schematic representation of drug treatment. (c) Survival of $P \operatorname{ten}{ }^{\Delta / \Delta}$ animals treated with vehicle (blue; $n=7 ;$ median survival $=23$ days), BYL719 (green; $n=8$; median survival $=32$ days), KIN193 (red; $n=7$; median survival $=50$ days), IC 87114 (orange; $n=7 ;$ median survival $=28$ days) and AS605240 (brown; $n=6$; median survival $=23$ days). ${ }^{\star} P<0.05$; ${ }^{\star \star} P<0.001$; ${ }^{\star \star \star} P<0.0001$. The log-rank test was applied to compare survival. (d) Spleen weight of vehicle-treated $(n=4)$ and KIN193-treated animals $(n=3)$ at the moribund stage. ${ }^{\star} P<0.05$. Student's $t$-test was applied. (e) Western blot analysis of Akt signalling in whole BM cells at 7 DPI. Freshly isolated BM cells were treated with DMSO, BYL719, KIN193, GS1101 or NVSPI35 at the $1-\mu \mathrm{M}$ dose for $2 \mathrm{~h}(n=6)$ for each treatment. (f) Phospho-flow analysis of p-Akt on isoform-selective inhibitor-treated animals. Freshly isolated lineage-negative BM cells were treated with inhibitors as in (e) and subjected to flow cytometry for LSK staining and intracellular P-Akt staining $(n=3)$ for each, and median fluorescence intensities were normalized to control.

potent chemo-attractant of stem cells that signals through CXCR4. Lineage-negative BM cells from $\operatorname{Pten}^{\Delta / \Delta}$ mice showed increased migration towards CXCL12 compared with WT control cells (Fig. 7d). This migration was abolished by the GPCR inhibitor pertussis toxin (PTX; Fig. 7d). Interestingly, we observed significantly reduced migration of $\operatorname{Pten}^{\Delta / \Delta} ; \mathrm{p} 110 \beta^{\Delta / \Delta}$ cells, and of Pten $^{\Delta / \Delta}$ cells treated with either KIN193 or NSC23766, compared with Pten ${ }^{\Delta / \Delta}$ and Pten ${ }^{\Delta / \Delta} ; p 110 \alpha^{\Delta / \Delta}$ cells (Fig. 7d). This suggests that deletion of $\mathrm{p} 110 \beta$, or pharmacologic inhibition of either p $110 \beta$ or Rac, partially interferes with the migration of $\operatorname{Pten}^{\Delta / \Delta}$ 



HA-RBD-mut P110ß $\quad-\quad+\quad-\quad-\quad-\quad+$

C

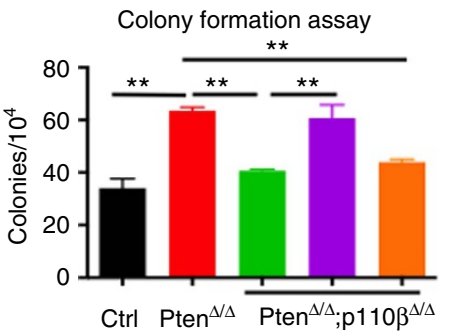

HA-wt P110ß $-\quad-\quad-\quad+\quad-$

d
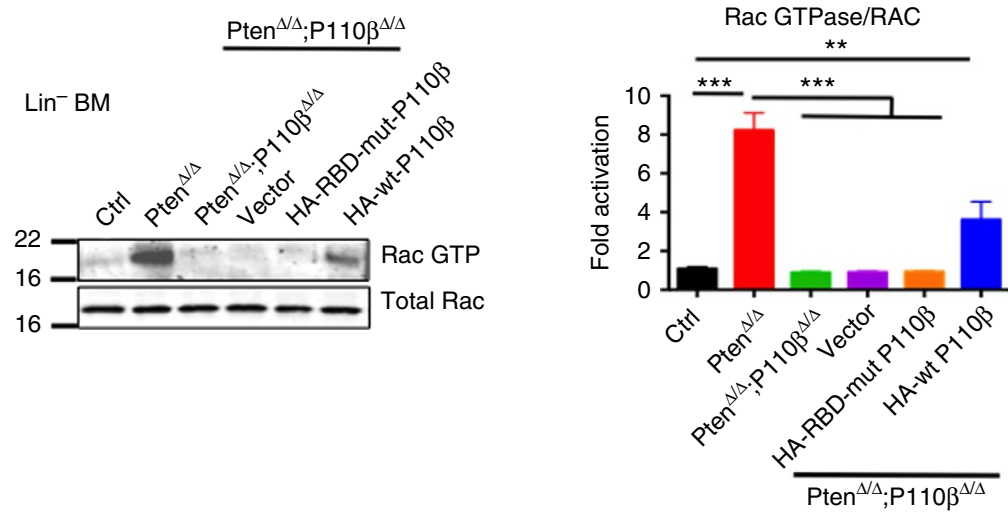

Figure 6 | Pten-deficient haematopoietic cells depend on the p110 the corresponding genotypes. Rac-GTP and total Rac were measured and normalized ( $n=3$; mean with s.e.m.). Two-way ANOVA test was applied to compare the RAC-GTPase/RAC levels. (b) Pten ${ }^{\Delta / 4} ;$ p $110 \beta^{\Delta / \Delta}$ BM cells were transduced with retroviruses expressing HA-tagged wild-type P110 $\beta$ or RBDmutant P110 $\beta$, and briefly selected in the presence of puromycin. HA immunoblotting was performed to show expression of RBD-mutant and wild-type $\mathrm{P} 110 \beta$ ( $n=3$ for each group). (c) Cells from WT control, $P \operatorname{ten}{ }^{\Delta / \Delta}$ or $P \operatorname{ten}{ }^{\Delta / \Delta} ; p 110 \beta^{\Delta / \Delta}$ animals were cultured in methylcellulose with myeloid growth factors in the presence of puromycin for 7 days, and colonies were counted at the end of day 7 ( $n=3$ for each group). Two-way ANOVA test was applied to compare the colony numbers. (d) Lineage-negative cells were isolated from WT control, Pten ${ }^{\Delta / \Delta}$ or Pten ${ }^{\Delta / \Delta} ;$ p $110 \beta^{\Delta / \Delta}$ mice, and the Rac-GTP activity assay was performed as in (a). Rac-GTP and total Rac were measured and normalized to WT ( $n=6$; mean with s.e.m.). Two-way ANOVA test was applied to compare the RAC-GTPase/RAC levels. ${ }^{\star \star} P<0.01,{ }^{\star \star \star} P<0.001$

cells towards a CXCL12 gradient, likely through perturbed GPCR signalling.

Because murine models of haematopoietic-specific Racl and Rac2 deficiency have revealed differential roles of Rac proteins in terms of HSPC function, we wanted to understand which Rac isoform is more important in the absence of Pten. To this end, we used siRNA to knockdown either Rac1 or Rac2, or both, and performed colony assays on $\operatorname{Pten}^{\Delta / \Delta}$ and $\operatorname{Pten}^{\Delta / \Delta} ; p 110 \beta^{\Delta / \Delta} \mathrm{BM}$ cells (Fig. 7e,f; Supplementary Fig. 10). We also tested the Rac inhibitor NSC23766, which targets both Rac1 and Rac2 (ref. 46). Knockdown of either Rac1 or Rac2, or their combined knockdown or pharmacological inhibition significantly reduced colony formation by $\operatorname{Pten}^{\Delta / \Delta}$ cells to levels obtained with $\operatorname{Pten}^{\Delta / \Delta}$; $p 110 \beta^{\Delta / \Delta}$ BM cells (Fig. 7f). However, knockdown of Rac1 or Rac2, the combination, or NSC23766 treatment did not further suppress colony formation beyond the effects of p110 $\beta$ deletion (Fig. 7f), suggesting that there is no additive or synergistic effect of Rac inhibition with $110 \beta$ deficiency in $\operatorname{Pten}^{\Delta / \Delta}$ cells. Together, these results suggest that $\mathrm{p} 110 \beta-\mathrm{Rac} 1 / 2$ work in concert to mediate the effects of Pten loss in promoting myeloid neoplasia.

\section{Discussion}

We and others have reported that Pten-deficient solid tumours frequently rely on p110 $\beta$ (refs $20,23,47$ ). In this study, we report for the first time an essential role for $\mathrm{p} 110 \beta$ in promoting haematologic neoplasia driven by Pten deletion in HSCs despite the expression of four different PI3K isoforms in haematopoietic cells. We have also found that $\mathrm{p} 110 \beta$ contributes to HSC depletion in the BM after Pten deletion. Interestingly, we found that Mx1Cre-mediated deletion of $\mathrm{p} 110 \beta$ in HSPCs of animals that are WT for Pten does not significantly affect blood counts. In fact, these animals appear healthy for many months after excision, suggesting that targeting p $110 \beta$ may lead to an effective therapy for myeloid leukaemia with little toxicity to normal HSCs.

Despite the marked impact of genetic deletion or pharmacologic inhibition of $\mathrm{p} 110 \beta$ on MPN, and the significantly delayed 
a

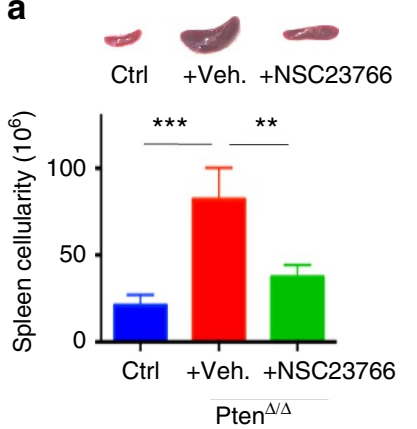

b

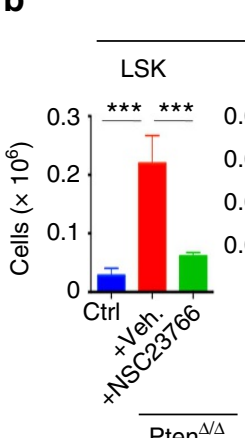

Spleen

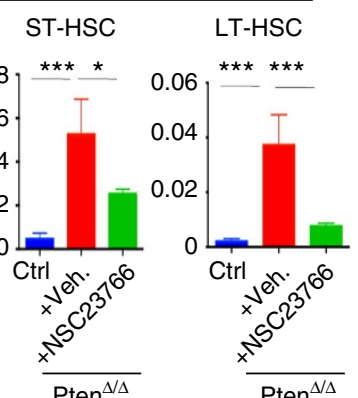

c

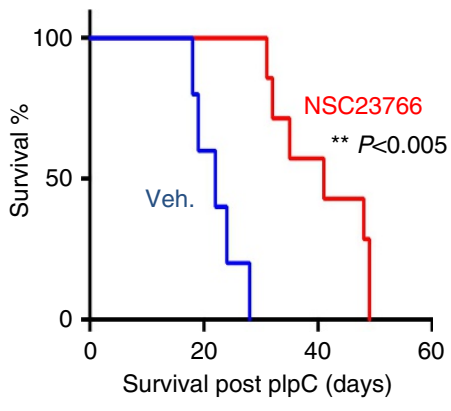

d

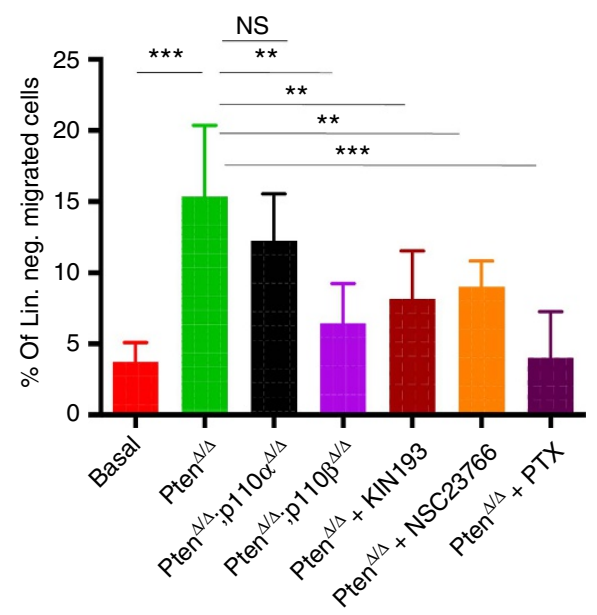

f

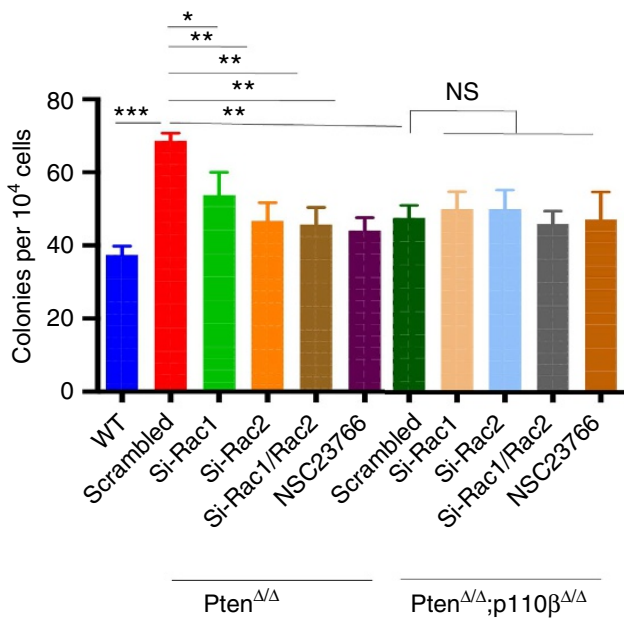

e
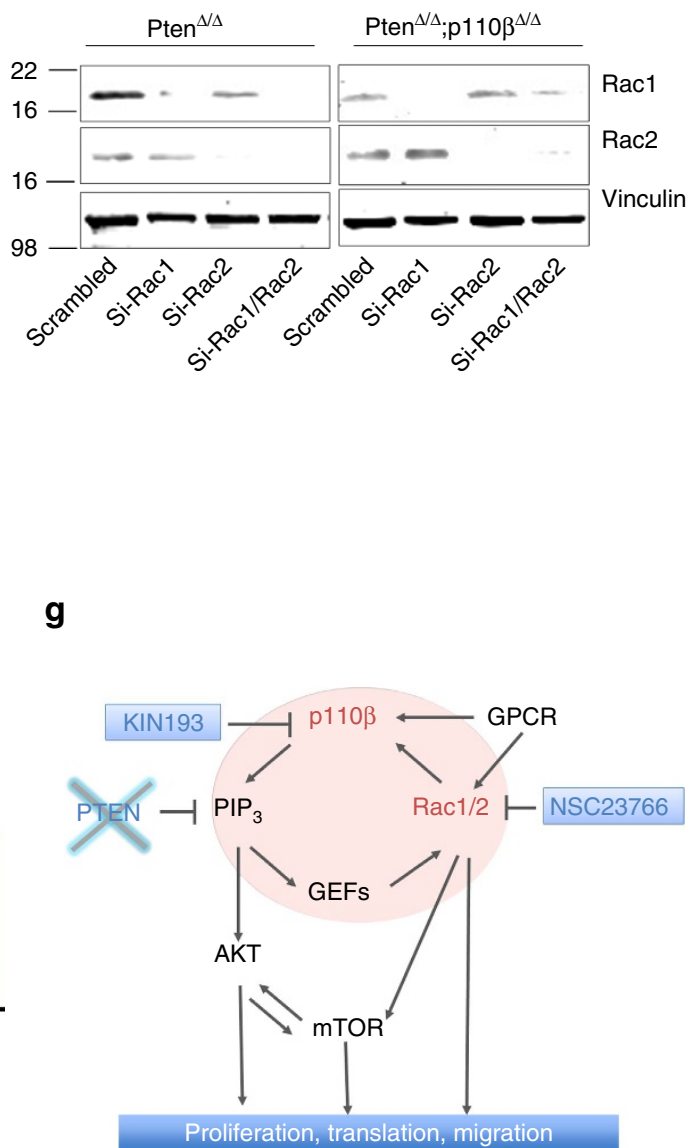

Figure 7 | Pten-deficient haematopoietic cells depend on the p110 $\beta$-Rac1 signalling axis. (a) NSC23766 was given at $2.5 \mu g g^{-1}$ daily intraperitoneally (IP). Spleen size and cellularity of vehicle-treated $(n=3)$ and NSC23766-treated $P \operatorname{ten}^{\Delta / \Delta}$ animals $(n=3)$ at 17 DPI. ${ }^{\star} P<0.05 ;{ }^{\star \star} P<0.001 ;{ }^{\star \star \star} P<0.0001$. Two-way ANOVA test was applied to compare spleen size. (b) Quantification of the numbers of LSK $\left(\mathrm{Lin}^{-} \mathrm{Sca}-1^{+} \mathrm{c}^{-} \mathrm{kit}^{+}\right), \mathrm{ST}^{-\mathrm{HSC}}\left(\mathrm{CD} 150^{-} \mathrm{CD} 48^{-} \mathrm{Lin}^{-}\right.$

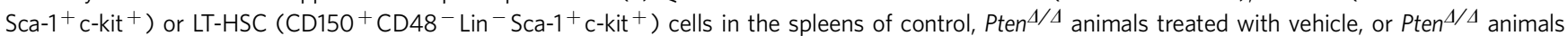
treated with NSC23766 at $17 \mathrm{DPI}$, determined by flow cytometry. The numbers represent total cell counts per spleen $\left(n=3 ; \mathrm{ctrl}, P\right.$ ten ${ }^{\Delta / \Delta}$ vehicle and NSC23766 treated). (c) Survival of $\operatorname{Pten}^{\Delta / \Delta}$ animals treated with vehicle (blue; $n=7$; median survival $=22$ days) or NSC23766 (red; $n=7 ;$ median survival $=42$ days). ${ }^{\star \star} P<0.005$. The log-rank test was applied to compare survival. (d) Lineage-negative BM cells were isolated from control, $P$ ten ${ }^{4 / \Delta}$ or $\operatorname{Pten}^{\Delta / \Delta}: P 110 \beta^{\Delta / \Delta}$ Pten $^{\Delta / \Delta}: P 110 \alpha^{\Delta / \Delta}$ on 7 DPI, and lineage-negative cells were pretreated for $3 \mathrm{~h}$ with KIN193, NSC23766 (50 $\mu$ M) or pertussis toxin (PTX; $100 \mathrm{ng} \mathrm{ml}^{-1}$ ). Migration towards the lower chamber (supplemented with CXCL12, $50 \mathrm{nM}$ ) was measured as the fraction of migrated cells relative to untreated Pten-null bone marrow-derived cells. Numbers of replicates are $n=4$ (basal, $\operatorname{Pten}^{\Delta / \Delta}$ no Cxcl12; Pten ${ }^{\Delta / \Delta}+$ PTX), $10\left(P \operatorname{ten}{ }^{\Delta / \Delta}\right), 6\left(P \operatorname{ten}{ }^{\Delta / \Delta}: P 110 \beta^{\Delta / \Delta}\right.$ ) and 6


with two different siRNAs targeting scrambled, mouse Rac1, mouse Rac2 alone, or both Rac1 and Rac2 and subjected to immunoblotting $48 \mathrm{~h}$ later. (f) Pten ${ }^{\Delta / \Delta}$ and Pten ${ }^{\Delta / \Delta} ;$ p110 $\beta^{\Delta / \Delta}$ BM cells from knockdown were transfected with two different siRNAs targeting scrambled, mouse Rac1, mouse Rac2 alone, or both Rac1 and Rac2, or treated with NSC23766 (50 $\mu \mathrm{M})$ and then colony assays were performed in methylcellulose with myeloid growth factors. Colonies were counted after 7 days $(n=3)$ for each group. Two-way ANOVA test was applied to calculate colony formation. (g) A working model of signalling cascades downstream of $\mathrm{P} 110 \beta$ and the signalling loop of $\mathrm{P} 110 \beta$ and Rac1. 
onset of leukaemia, a fraction of $\operatorname{Pten}^{\Delta / \Delta} ; p 110 \beta^{\Delta / \Delta}$ animals succumbed to T-ALL at a later time. Berenjeno et al. ${ }^{48}$ showed that in Pten ${ }^{+/-}$animals, inactivation of $\mathrm{p} 110 \beta$ led to reduced PIP3 generation in lymphoma tissues, but had little impact on lymphoma formation. It is possible that these tumours become $\mathrm{p} 110 \beta$ independent through the acquisition of secondary alterations. In fact, Yilmaz et al. ${ }^{10}$ have documented the presence of cytogenetic alterations in leukaemic blasts from Pten ${ }^{4 / \Delta}$ animals. Alternatively, isoform dependency may shift with cell differentiation. For example, the isoform dependency in the skin hamartoma driven by Pten loss changed from a p110 $\beta$ dependency in the basal layer of the epidermis to a p110 $\alpha$ dependency in the suprabasal cells as the basal cells underwent stepwise differentiation to become suprabasal cells ${ }^{37}$. In this study, we also provide evidence that neither $\mathrm{p} 110 \alpha$ nor $\mathrm{p} 110 \beta$ has any effect on T-ALL driven by T lymphocyte-specific deletion of Pten using Lck-Cre. In this system, it has been shown that $\mathrm{p} 110 \delta$ and $110 \gamma$ contribute to T-ALL induced by Pten loss in T cells ${ }^{28}$. These studies provide additional data that accentuate the distinct roles of $\mathrm{p} 110 \beta$ in the HSCs and in myeloid and lymphoid tumour initiation in the absence of Pten.

Interestingly, we found that inhibition of $\mathrm{p} 110 \beta, \mathrm{p} 110 \alpha$ or p110 could similarly reduce p-Akt in Pten-deficient BM and HSCs, suggesting an Akt-independent pathway specific to p110 $\beta$ is important in Pten-deficient HSCs in promoting myeloid neoplasia. We report a new mechanistic insight that may explain the unique role of $p 110 \beta$ in this setting. Since $p 110 \beta$ binds to Rac rather than to Ras via its RBD, unlike the other class I PI3K isoforms ${ }^{21}$, we investigated the role of the p110 $\beta$-Rac axis in the setting of myeloid neoplasia induced by Pten loss in HSCs. Notably, Rac signalling is not only important for the activation of p110 $\beta$ but it itself is also activated by PIP3 via PIP3-activated guanine-nucleotide exchange factors, forming a potential signalling loop (Fig. $7 \mathrm{~g})^{14,49}$. We found strong evidence that this loop is indeed active in our Pten-deficient model. We show that Rac was activated in Pten-null BM cells, and this activation was suppressed in Pten/p110 $\beta$ double KOs, but not in $\mathrm{Pten} / \mathrm{p} 110 \alpha$ double KOs. This hypothesis was further supported by our finding that only wild-type $\mathrm{p} 110 \beta$, but not the RBD mutant of $\mathrm{p} 110 \beta$, rescued colony formation in Pten $/ \mathrm{p} 110 \beta$ deficient BM cells. Notably, the effect of the RBD-mutant p110 $\beta$ on inhibiting colony formation in Pten-null BM cells is comparable to that of $\mathrm{p} 110 \beta$ deletion. An intact RBD was reported to be required for membrane localization of $110 \beta$ for both signalling and oncogenic transformation by wild-type p1 $10 \beta$ in cultured cells ${ }^{40}$. Our data suggest that the interaction of p110 $\beta$-Rac may play an important role in mediating p110 $\beta$ activity downstream of GPCRs and tyrosine kinases in the context of Pten deficiency. Moreover, pharmacologic inhibition of $\mathrm{p} 110 \beta$ or Rac in Pten-deficient mice resulted in a strikingly similar functional rescue in vivo, with a reduction in extramedullary haematopoiesis in the spleen and improved survival.

Of the three isoforms of Rac family GTPases, Rac2 is expressed specifically in haematopoietic cells, while Rac1 and Rac3 are ubiquitously expressed ${ }^{44,50}$. The Rac inhibitor NSC23766 targets all three Rac members: Rac1, 2 and 3 (ref. 46). Both Rac1 and CDC42 have been shown to bind $\mathrm{p} 110 \beta$ in a recent study ${ }^{21}$. Binding to Rac2/3 was not tested, but might also be expected, based on the homology of their effector domains with that of Rac1. Previous studies have suggested that Rac1 and Rac2 play both distinct and overlapping roles in HSCs and progenitor cells, while the role of Rac3 in haematopoiesis has not been defined $^{44,51}$. It also has been shown that targeting both Rac1 and Rac2 was effective in a mouse model of BCR-ABL-induced MPN, as well as in a mouse model of MLL-AF9 AML ${ }^{46,52}$. Interestingly, our data show that the effect of knockdown of Rac2 is comparable to that of combined knockdown of both Racl and Rac2, or a pan-Rac inhibitor NSC23766. Since Rac2 is primarily expressed in haematopoietic cells, our data suggest that Rac2 could potentially be a better pharmacologic target with reduced toxicity.

Yilmaz et al. have shown that the mTOR inhibitor rapamycin can rescue HSC depletion and can suppress the development of leukaemia in vivo. More recently, the Armstrong and Morrison groups reported that $\mathrm{mTORC} 1$ and $\mathrm{mTORC} 2$ play critical roles in haematopoiesis and Pten-loss-driven leukaemogenesis, respectively ${ }^{53,54}$. By ablation or inhibition of p110 $\beta$, we obtained similar results suggesting that the activation of mTOR in HSCs by Pten loss may be mediated largely by $\mathrm{p} 110 \beta$. A recent study demonstrated that Racl regulates the activity of both mTORC1 and mTORC2 (ref. 55), providing a potential Akt-independent link between $\mathrm{p} 110 \beta-\mathrm{Rac}$ and mTOR. Together, these data suggest that p110 $3-$ Rac acts upstream of Akt and mTOR. We feel that our data are most consistent with the working model shown in Fig. $7 \mathrm{~g}$, in which p110 $\beta$ works in a signalling loop with Rac to generate the key signals arising from Pten loss. Notably, these signals include both Akt-dependent and Akt-independent pathways leading to cell proliferation and migration.

In summary, our results provide the first evidence that PI3K-p110 $\beta$ plays an essential role in controlling HSC function in the setting of Pten loss ${ }^{56}$. We have also uncovered a specific role for $\mathrm{p} 110 \beta$ in myeloid leukaemia induced by Pten deficiency. In contrast, we found that $\mathrm{p} 110 \beta$ is dispensable for T-ALL induced by Pten loss. Most importantly, our data show that a p110 $\beta$-Rac signalling loop is important for the induction of myeloid neoplasia in the absence of Pten. Thus, secondgeneration p110 $\beta$ - or Rac-selective inhibitors may interrupt this loop, thereby providing a promising new therapeutic strategy for Pten-deficient myeloid leukaemias while preserving normal haematopoiesis.

\section{Methods}

Animal experiments. Mice were housed in a pathogen-free animal facility at Dana-Farber Cancer Institute (DFCI). All animal experiments conformed to the relevant regulatory standards, guidelines and regulations, and were approved by the DFCI-Institutional Animal Care and Use Committee (IACUC). Dead and moribund animals were included in survival curves. Pten ${ }^{\text {fl/fl }}$ mice (obtained from Hong Wu's Laboratory) were crossed with $\mathrm{Pten}^{\mathrm{fl} /+}$ animals carrying an Mx-1-Cre transgene (obtained from Dr Gary Gilliland's laboratory) to obtain Mx-1-Cre ${ }^{+}$; Pten $^{\mathrm{fl} / \mathrm{fl}}$ mice $\left(\right.$ Pten $\left.^{\Delta / \Delta}\right)$. p110 $\alpha$ and p110 $\beta$ mutants were obtained by crossing $\mathrm{Mx}-1-\mathrm{Cre}^{+} ; \mathrm{Pten}^{\mathrm{fl} /}+$ mice with $\mathrm{p} 110 \alpha^{\mathrm{fl} / \mathrm{fl}}, \mathrm{p} 110 \beta^{\mathrm{fl} / \mathrm{fl}}$ animals to obtain Mx-1-Cre ${ }^{+}$; Pten $^{\mathrm{fl} / \mathrm{fl}} ; \mathrm{p} 110 \alpha^{\mathrm{fl} / \mathrm{fl}}\left(\right.$ Pten $\left.^{\Delta / \Delta} ; \mathrm{p} 110 \alpha^{\Delta / \Delta}\right)$ and $\mathrm{Mx}-1-\mathrm{Cre}^{+} ; \mathrm{Pten}^{\mathrm{fl} / \mathrm{fl}} ; \mathrm{p} 110 \beta^{\mathrm{fl} / \mathrm{fl}}$ animals $\left(\right.$ Pten $\left.^{\Delta / \Delta} ; p 110 \beta^{\Delta / \Delta}\right)$. P $110 \delta$ germline knockout animals (James Ihle's laboratory) were backcrossed to the C57BL/6 background for more than nine generations and then crossed with $\mathrm{Mx}-1-\mathrm{Cre}^{+} ; \mathrm{Pten}^{\mathrm{fl} /+}$ to obtain $\mathrm{Mx}-1-\mathrm{Cre}^{+} ; \mathrm{Pten}^{\mathrm{f} / \mathrm{fl}} ; \mathrm{p} 110 \delta^{-/-}$ animals $\left(\right.$ Pten $\left.^{\Delta / \Delta} ; p 110 \delta^{-/-}\right)$. Thirty day-old male and female animals were induced by intraperitoneal injection with $\mathrm{pIpC}\left(25 \mathrm{mg} \mathrm{g}^{-1}\right.$; GE Healthsciences $)$ every other day for 5 days. WT;Mx-1-Cre ${ }^{+}$mice were used as controls for all experiments. Peripheral blood was obtained by retro-orbital venous blood sampling. Peripheral blood counts were analysed on a Hemavet 950FS blood analyzer (Drew Scientific).

Flow cytometry. All data acquisition was performed on a LSRII (BD) flow cytometer, and results were analysed using FlowJo v.8.8.7 (Tree Star). Antibodies used for flow cytometry were directly coupled and directed against B220 (APC, BD Pharmingen), cKit (PE-Cy7, BioLegend), CD3 (PE-Cy7, BD Bioscience), CD4 (APC-H7, BD Pharmingen), CD8 (ECD, Beckman Coulter), CD11b (PE, BD Bioscience), CD16/32 (PE, eBioscience), CD34 (FITC, BD Pharmingen), CD45.1 (FITC, BD Pharmingen), CD45.2 (PerCP-Cy5.5, BD Pharmingen), CD48 (APC-Cy7, BD Pharmingen), CD127 (ECD, BD Pharmingen), CD150 (PerCP-Cy5.5, BioLegend), Gr1 (APC-Alexa 700, BD Bioscience), lineage cocktail (APC, BD Pharmingen), Scal (Brilliant Violet 421, BioLegend), Ki67 (Alexa 488, BD Pharmingen). Dead cells were excluded using either DAPI or Vivid-Aqua (Invitrogen) staining

Chemotaxis assays. Fresh BM cells were isolated from mutant mice and from control animals, and lineage-positive cells were depleted using magnetic separation 
(lineage depletion kit, Miltenyi Biotec) and were resuspended in PBS containing $0.5 \%$ BSA. Lineage-negative cells $\left(0.5-1\right.$ million) were pretreated for $3 \mathrm{~h}$ at $37^{\circ} \mathrm{C}$ with the p110ß-specific inhibitor KIN193 $(1 \mu \mathrm{M})$, NSC23766 $(50 \mu \mathrm{M})$ PTX from Bordetella pertussis $\left(100 \mathrm{ng} \mathrm{ml}^{-1}\right.$ ) or dimethylsulphoxide (DMSO; 1:1,000), or directly loaded $\left(10^{5}\right.$ cells in $\left.100 \mu \mathrm{l}\right)$ in the top chamber of a $6.5-\mathrm{mm}$ diameter $8-\mu \mathrm{m}$ pore polycarbonate Transwell insert (Corning Costar); inserts with cells were then placed in wells containing CXCL12 (50 mM, PeproTech), and incubated for $3 \mathrm{~h}$ at $37^{\circ} \mathrm{C}$. Cells migrating from the top to the bottom chamber were collected and quantified using a Countess cell counting machine (Invitrogen).

Compounds. TGX221 was obtained from Chemdea; NSC23766 and IC87114 from Selleck Chemicals; GDC0941 from MedChemexpress; KIN193 from Haoyuan Chemexpress; NVP-BYL719, NVSPI35, AS605240 and IC87114 were synthesized by Chemitek (Indianapolis, USA). Mice were dosed once by intraperitoneal injection of KIN193 $\left(20 \mathrm{mg} \mathrm{kg}^{-1}\right)$ formulated in 7.5\% NMP (Sigma-Aldrich), $40 \%$ polyethylene glycol 400 (Sigma-Aldrich) and $52.5 \% \mathrm{dH}_{2} \mathrm{O}$; once by intraperitoneal injection of NSC23766 $\left(2.5 \mathrm{mg} \mathrm{kg}^{-1}\right)$, or gavaged once daily with NVP-BYL719 $\left(45 \mathrm{mg} \mathrm{kg}^{-1}\right)$ in $0.5 \%$ methylcellulose; AS605240 $\left(50 \mathrm{mg} \mathrm{kg}^{-1}\right)$ in $0.5 \%$ carboxymethylcellulose/0.25\% Tween-20; or IC87114 (45 $\left.\mathrm{mg} \mathrm{kg}^{-1}\right)$ dissolved in $75 \%$ polyethylene glycol.

Colony-forming assays. BM and spleen cells were collected, subjected to red-cell lysis and resuspended in Iscove's modified Dulbecco's medium $/ 10 \%$ fetal bovine serum $/ 5 \%$ penicillin-streptomycin. Cells were plated in the presence of inhibitors in duplicate in M3434 methylcellulose media (Stemcell Technologies) at $1 \times 10^{4}$ cells per dish for BM and $5 \times 10^{4}$ cells per dish for spleen cells. Colonies were scored after 7 days.

Rac activation assay. BM cells from corresponding mice at 7 DPI of pIpC were collected and immediately subjected to Racl activation assay with the Racl activation assay kit (Millipore) according to the manufacturer's instructions.

Western blotting. Whole-cell protein lysates were prepared from single-cell suspensions of BM and splenocytes from corresponding mice, and from agematched WT C57 Bl/6 mice. Western blotting was performed with the slight changes as follows: $0.45-\mu \mathrm{M}$ nitrocellulose membranes (Millipore) were used to transfer the proteins, this followed by blocking $1 \mathrm{~h}$ at room temperature in $1 \times \mathrm{TBS}$, $5 \%$ nonfat milk. Primary antibodies used were as follows: p110 $\alpha$ (1:1,000, catalogue no. 4249; Cell Signaling), p110 $\beta$ (1:1,000, catalogue no. sc-602; Santa Cruz), p110 $\delta$ (1:1,000, catalogue no. sc-55589; Santa Cruz), Akt (1:1,000, catalogue no. 9272; Cell Signaling), phospho-Akt (Ser473; 1:500, catalogue no. 4050; Cell Signaling), S6 (1:1,000, catalogue no. 2217; Cell Signaling), phospho-S6 (catalogue no. 2211; Cell Signaling), Rac1 (1:500, catalogue no. 05-389; Millipore), Rac2 (1:500, catalogue no. sc-96; Santa Cruz), $\alpha$-HA (1:2,000 catalogue no. H6908; Sigma-Aldrich), $\alpha$-tubulin (1:5,000 catalogue no. T9026; Sigma-Aldrich) and vinculin (1:5,000 catalogue no. sc-73614, Santa Cruz). The directly fluorophore-conjugated secondary antibodies: anti-mouse 680 and anti-rabbit 800 (LICOR) were used at dilutions of 1:5,000. LICOR Odyssey instrument was used to develop the membranes. Signal intensity with background correction was quantified using LICOR Image Studio software. Whole Western Blot pictures are shown in Supplementary Fig. 10.

SiRNA knockdown. Two separate SiRNAs, targeting mouse Racl (J-041170-050002 and J-041170-06-0002) and Rac2 (J-041171-09-0002 and J-041171-10-0002) were ordered from Dharmacon (Dharmacon, GE Healthcare) transfected in Invitrogen X15 media to freshly isolated whole bone marrow (WBM) cells using RNAiMAX (Invitrogen) in 10-cm plates according to the manufacturer's instructions. Forty-eight hours later after transfection, cells were subjected to immunoblotting using Rac1 or Rac2 antibodies or colony-forming experiments.

Histology. Recipient mice were killed at the indicated time points, or when they began to show signs of disease. Organs were fixed in formalin, and histology slides were prepared and stained at the Brigham and Women's Rodent Histology Core Facility. Digital images were acquired on a Nikon Eclipse E400 microscope equipped with a digital camera and analysed using Spot Advanced software.

Immunohistochemistry. For histological analyses, formalin-fixed tissue sections were embedded in paraffin, sectioned and stained with haematoxylin and eosin by the Dana-Farber/Harvard Cancer Center Rodent Histopathology Core.

P-Akt flow cytometry analysis of LSK cells. Phospho-flow cytometry was performed as previously described ${ }^{56}$, with the following modifications: lineagenegative $\mathrm{BM}$ cells from mutant animals were isolated using lineage depletion kit (Miltenyi Biotec) and serum starved for $1 \mathrm{~h}$, and treated for $2 \mathrm{~h}$ with $1 \mu \mathrm{M}$ of BYL719, KIN193, GS1101 and NVSPI3. Cells were then fixed with 4\% paraformaldehyde, and permeabilized with cold $100 \%$ acetone. Cells were than stained simultaneously with c-kit, Sca-1 and anti-mouse P-Akt (Alexa 647; 1:100 dilution, cat. no. 2337, Cell Signaling). All data acquisition was performed on a LSRII (BD) flow cytometer, and results were analysed and basal level of P-Akt was calculated as normalized to WT cells by calculating median fluorescent intensity using FlowJo v.8.8.7 (Tree Star).

Long-term competitive repopulation assays. Recipient mice (4-6 weeks old female mice; B6.SJL strain) received two doses of 540 rads each, delivered $3 \mathrm{~h}$ apart. Nucleated BM cells from control and Pten ${ }^{\Delta / 4}$ mice $(\mathrm{C} 57 \mathrm{Bl} / 6)$ or from compound

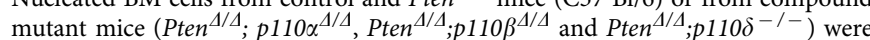
mixed with wild-type competitor BM-nucleated cells (B6.SJL), and were injected into the retro-orbital venous sinus of irradiated recipients. Peripheral blood was obtained retro-orbitally every 4 weeks, subjected to red-cell lysis and analysed by flow cytometry to assess donor cell engraftment for up to 20 weeks after transplantation. Pilot experiments showed that Pten-mutant animals had a greatly reduced repopulation capacity; therefore, an excess of mutant cells over control cells was used. Each recipient mouse received $1 \times 10^{6}$ Pten mutant, ctrl or compound mutant BM-nucleated cells plus $2 \times 10^{5}$ competitor cells. Tissues from recipient mice were collected and stained for pathological examination.

Plasmid constructs. Mutations were generated in the pBabe-HA wild-type P110 $\beta$ by site-directed mutagenesis to obtain p110 $\beta-S 205 \mathrm{D} / \mathrm{K} 224 \mathrm{~A}$ double mutant to obtain Ras-binding mutant $\mathrm{P} 110 \beta$. This plasmid was used to transduce $P \operatorname{Pten}{ }^{\Delta / \Delta}$; $p 110 \beta^{\Delta / \Delta} \mathrm{BM}$ cells and for colony-forming experiments.

Statistical methods. Two-tailed $T$-tests (two groups) and analysis of variance (ANOVA) tests (multiple groups) and log-rank statistics (Mantel-Cox test; survival studies) were used, and calculated with the use of Prism 6.0 (GraphPad). Data are represented as mean \pm s.d. unless otherwise noted. ${ }^{*} P<0.05 ;{ }^{*} P<0.01$; ${ }^{* * *} P<0.001$

\section{References}

1. Martelli, A. M. et al. Phosphoinositide 3-kinase/Akt signaling pathway and its therapeutical implications for human acute myeloid leukemia. Leukemia 20, 911-928 (2006).

2. Peng, C. et al. PTEN is a tumor suppressor in CML stem cells and BCR-ABL-induced leukemias in mice. Blood 115, 626-635 (2010).

3. Gutierrez, A. et al. High frequency of PTEN, PI3K, and AKT abnormalities in T-cell acute lymphoblastic leukemia. Blood 114, 647-650 (2009).

4. Dahia, P. L. et al. PTEN is inversely correlated with the cell survival factor $\mathrm{Akt} / \mathrm{PKB}$ and is inactivated via multiple mechanismsin haematological malignancies. Hum. Mol. Genet. 8, 185-193 (1999).

5. Patel, J. P. et al. Prognostic relevance of integrated genetic profiling in acute myeloid leukemia. N. Engl. J. Med. 366, 1079-1089 (2012).

6. Li, Y. et al. Epigenetic silencing of microRNA-193a contributes to leukemogenesis in $\mathrm{t}(8 ; 21)$ acute myeloid leukemia by activating the PTEN/PI3K signal pathway. Blood 121, 499-509 (2013).

7. Noguera, N. I. et al. Nucleophosmin/B26 regulates PTEN through interaction with HAUSP in acute myeloid leukemia. Leukemia 27, 1037-1043 (2013).

8. Yang, J. et al. A reappraisal by quantitative flow cytometry analysis of PTEN expression in acute leukemia. Leukemia 21, 2072-2074 (2007).

9. Xu, Q., Simpson, S. E., Scialla, T. J., Bagg, A. \& Carroll, M. Survival of acute myeloid leukemia cells requires PI3 kinase activation. Blood 102, 972-980 (2003).

10. Yilmaz, O. H. et al. Pten dependence distinguishes haematopoietic stem cells from leukaemia-initiating cells. Nature 441, 475-482 (2006).

11. Zhang, J. et al. PTEN maintains haematopoietic stem cells and acts in lineage choice and leukaemia prevention. Nature 441, 518-522 (2006).

12. Tang, P. et al. Differential roles of Kras and Pten in murine leukemogenesis. Leukemia 27, 1210-1214 (2013).

13. Vardiman, J. W. et al. The 2008 revision of the World Health Organization (WHO) classification of myeloid neoplasms and acute leukemia: rationale and important changes. Blood 114, 937-951 (2009).

14. Thorpe, L. M., Yuzugullu, H. \& Zhao, J. J. PI3K in cancer: divergent roles of isoforms, modes of activation and therapeutic targeting. Nat. Rev. Cancer 15, 7-24 (2014).

15. Utermark, T. et al. The p110alpha and p110beta isoforms of PI3K play divergent roles in mammary gland development and tumorigenesis. Genes Dev. 26, 1573-1586 (2012).

16. Castellano, E. et al. Requirement for interaction of PI3-kinase p110alpha with RAS in lung tumor maintenance. Cancer Cell 24, 617-630 (2013).

17. Gritsman, K. et al. Hematopoiesis and RAS-driven myeloid leukemia differentially require PI3K isoform p110alpha. J. Clin. Invest. 124, 1794-1809 (2014).

18. Ciraolo, E. et al. Phosphoinositide 3-kinase p110beta activity: key role in metabolism and mammary gland cancer but not development. Sci. Signal. 1, ra3 (2008). 
19. Guillermet-Guibert, J. et al. The pl10beta isoform of phosphoinositide 3-kinase signals downstream of $\mathrm{G}$ protein-coupled receptors and is functionally redundant with pllogamma. Proc. Natl Acad. Sci. USA 105, 8292-8297 (2008).

20. Jia, S. et al. Essential roles of PI(3)K-p110beta in cell growth, metabolism and tumorigenesis. Nature 454, 776-779 (2008).

21. Fritsch, R. et al. RAS and RHO families of GTPases directly regulate distinct phosphoinositide 3-kinase isoforms. Cell 153, 1050-1063 (2013).

22. Ni, J. et al. Functional characterization of an isoform-selective inhibitor of PI3K-p110beta as a potential anticancer agent. Cancer Discov. 2, 425-433 (2012).

23. Wee, S. et al. PTEN-deficient cancers depend on PIK3CB. Proc. Natl Acad. Sci. USA 105, 13057-13062 (2008).

24. Furman, R. R. et al. Idelalisib and rituximab in relapsed chronic lymphocytic leukemia. N. Engl. J. Med. 370, 997-1007 (2014).

25. Zhao, J. J. et al. The p110alpha isoform of PI3K is essential for proper growth factor signaling and oncogenic transformation. Proc. Natl Acad. Sci. USA 103, 16296-16300 (2006).

26. Jou, S. T. et al. Essential, nonredundant role for the phosphoinositide 3-kinase p110delta in signaling by the B-cell receptor complex. Mol. Cell. Biol. 22, 8580-8591 (2002).

27. Kuhn, R., Schwenk, F., Aguet, M. \& Rajewsky, K. Inducible gene targeting in mice. Science 269, 1427-1429 (1995).

28. Subramaniam, P. S. et al. Targeting nonclassical oncogenes for therapy in T-ALL. Cancer Cell 21, 459-472 (2012).

29. Hagenbeek, T. J. \& Spits, H. T-cell lymphomas in T-cell-specific Pten-deficient mice originate in the thymus. Leukemia 22, 608-619 (2008).

30. Hagenbeek, T. J. et al. The loss of PTEN allows TCR alphabeta lineage thymocytes to bypass IL-7 and Pre-TCR-mediated signaling. J. Exp. Med. 200, 883-894 (2004).

31. Tesio, M. et al. Pten loss in the bone marrow leads to G-CSF-mediated HSC mobilization. J. Exp. Med. 210, 2337-2349 (2013).

32. Juric, D. et al. Phase I study of BYL719, an alpha-specific PI3K inhibitor, in patients with PIK3CA mutant advanced solid tumors: preliminary efficacy and safety in patients with PIK3CA mutant ER-positive $(\mathrm{ER}+)$ metastatic breast cancer (MBC). Cancer Res. 72, P6-10-07 (2012).

33. Lannutti, B. J. et al. CAL-101, a p110delta selective phosphatidylinositol-3kinase inhibitor for the treatment of B-cell malignancies, inhibits PI3K signaling and cellular viability. Blood 117, 591-594 (2011).

34. Bruce, I. et al. Development of isoform selective PI3-kinase inhibitors as pharmacological tools for elucidating the PI3K pathway. Bioorg. Med. Chem. Lett. 22, 5445-5450 (2012).

35. Sujobert, P. et al. Essential role for the p110delta isoform in phosphoinositide 3-kinase activation and cell proliferation in acute myeloid leukemia. Blood 106, 1063-1066 (2005).

36. Camps, M. et al. Blockade of PI3Kgamma suppresses joint inflammation and damage in mouse models of rheumatoid arthritis. Nat. Med. 11, 936-943 (2005).

37. Wang, Q. et al. Spatially distinct roles of class Ia PI3K isoforms in the development and maintenance of PTEN hamartoma tumor syndrome. Genes Dev. 27, 1568-1580 (2013).

38. Ali, K. et al. Essential role for the p110delta phosphoinositide 3-kinase in the allergic response. Nature 431, 1007-1011 (2004).

39. Kang, J. H. et al. Phosphorylation of Rho-associated kinase (Rho-kinase/ ROCK/ROK) substrates by protein kinases A and C. Biochimie 89, 39-47 (2007).

40. Denley, A., Kang, S., Karst, U. \& Vogt, P. K. Oncogenic signaling of class I PI3K isoforms. Oncogene 27, 2561-2574 (2008).

41. Klarlund, J. K. et al. Signaling by phosphoinositide-3,4,5-trisphosphate through proteins containing pleckstrin and Sec7 homology domains. Science 275, 1927-1930 (1997).

42. Welch, H. C. et al. P-Rex1, a PtdIns(3,4,5)P3- and Gbetagamma-regulated guanine-nucleotide exchange factor for Rac. Cell 108, 809-821 (2002).

43. Cancelas, J. A. et al. Rac GTPases differentially integrate signals regulating hematopoietic stem cell localization. Nat. Med. 11, 886-891 (2005).

44. Gu, Y. et al. Hematopoietic cell regulation by Rac1 and Rac2 guanosine triphosphatases. Science 302, 445-449 (2003).

45. Gao, Y., Dickerson, J. B., Guo, F., Zheng, J. \& Zheng, Y. Rational design and characterization of a Rac GTPase-specific small molecule inhibitor. Proc. Natl Acad. Sci. USA 101, 7618-7623 (2004).
46. Thomas, E. K. et al. Rac guanosine triphosphatases represent integrating molecular therapeutic targets for BCR-ABL-induced myeloproliferative disease Cancer Cell 12, 467-478 (2007).

47. Schwartz, S. et al. Feedback suppression of PI3Kalpha signaling in PTEN-mutated tumors is relieved by selective inhibition of PI3Kbeta. Cancer Cell 27, 109-122 (2015).

48. Berenjeno, I. M. et al. Both p110alpha and p110beta isoforms of PI3K can modulate the impact of loss-of-function of the PTEN tumour suppressor. Biochem. J. 442, 151-159 (2012).

49. Weiner, O. D. et al. A PtdInsP(3)- and Rho GTPase-mediated positive feedback loop regulates neutrophil polarity. Nat. Cell Biol. 4, 509-513 (2002).

50. Shirsat, N. V., Pignolo, R. J., Kreider, B. L. \& Rovera, G. A member of the ras gene superfamily is expressed specifically in T, B and myeloid hemopoietic cells. Oncogene 5, 769-772 (1990).

51. Yang, F. C. et al. Rac and Cdc42 GTPases control hematopoietic stem cell shape, adhesion, migration, and mobilization. Proc. Natl Acad. Sci. USA 98 5614-5618 (2001).

52. Mizukawa, B. et al. Inhibition of Rac GTPase signaling and downstream prosurvival $\mathrm{Bcl}-2$ proteins as combination targeted therapy in MLL-AF9 leukemia. Blood 118, 5235-5245 (2011).

53. Kalaitzidis, D. et al. mTOR complex 1 plays critical roles in hematopoiesis and Pten-loss-evoked leukemogenesis. Cell Stem Cell 11, 429-439 (2012).

54. Magee, J. A. et al. Temporal changes in PTEN and mTORC2 regulation of hematopoietic stem cell self-renewal and leukemia suppression. Cell Stem Cell 11, 415-428 (2012)

55. Saci, A., Cantley, L. C. \& Carpenter, C. L. Racl regulates the activity of mTORC1 and mTORC2 and controls cellular size. Mol. Cell. 42, 50-61 (2011)

56. Kalaitzidis, D. \& Neel, B. G. Flow-cytometric phosphoprotein analysis reveals agonist and temporal differences in responses of murine hematopoietic stem/progenitor cells. PLoS ONE 3, e3776 (2008).

\section{Acknowledgements}

We thank Drs E. Weisberg, Jhaveri-Schneider and Omer Yilmaz for scientific discussions and critical reading. We thank Dr J. Ihle (St. Jude Children's Research Hospital) for providing the $\mathrm{p} 110 \delta \mathrm{KO}$ mice. This work was supported by the Swiss National Science Foundation (FNSNF \#PBLA03-140100 to L.B.) and the Leukemia \& Lymphoma Society (LLS \#5408-13 to L.B.), and NIH grants 5K08CA149208 (K.G.), CA172461 (J.J.Z.) and CA187918 (T.M.R. and J.J.Z.).

\section{Author contributions}

H.Y., L.B., T.M.R., K.G. and J.J.Z contributed to conception and design; development of methodology; analysis and interpretation of data; and writing, review and/or revision of the manuscript. H.Y., L.B., T.V., H.T. and K.G. contributed to acquisition of data. T.V., A.S., H.T., J.N., L.K.C. and J.J.Z. were responsible for administrative, technical or material support. T.M.R., K.G. and J.J.Z. contributed to study supervision. T.V., A.S. and R.B. provided technical assistance.

\section{Additional information}

Supplementary Information accompanies this paper at http://www.nature.com/ naturecommunications

Competing financial interests: T.M.R. is a consultant/advisory board member of Novartis. The remaining authors declare no competing financial interests.

Reprints and permission information is available online at http://npg.nature.com/ reprintsandpermissions/

How to cite this article: Yuzugullu, H. et al. A PI3K p110 $\beta$-Rac signalling loop mediates Pten-loss-induced perturbation of haematopoiesis and leukaemogenesis. Nat. Commun. 6:8501 doi: 10.1038/ncomms9501 (2015).

This work is licensed under a Creative Commons Attribution 4.0 International License. The images or other third party material in this article are included in the article's Creative Commons license, unless indicated otherwise in the credit line; if the material is not included under the Creative Commons license, users will need to obtain permission from the license holder to reproduce the material. To view a copy of this license, visit http://creativecommons.org/licenses/by/4.0/ 